\title{
The estimation of truncation error by $\tau$-estimation revisited
}

\author{
F. Fraysse*, J. de Vicente, E. Valero \\ E.T.S.I. Aeronáuticos, Universidad Politécnica de Madrid, Ciudad Universitaria, E-28040 Madrid, Spain
}

A R T I C L E I N F O

\section{Keywords:}

Truncation error

Finite volume solvers

Multigrid

Uncertainty estimator

\begin{abstract}
A B S T R A C T
The aim of this paper was to accurately estimate the local truncation error of partial differential equations, that are numerically solved using a finite difference or finite volume approach on structured and unstructured meshes. In this work, we approximated the local truncation error using the $\tau$-estimation procedure, which aims to compare the residuals on a sequence of grids with different spacing. First, we focused the analysis on one-dimensional scalar linear and non-linear test cases to examine the accuracy of the estimation of the truncation error for both finite difference and finite volume approaches on different grid topologies. Then, we extended the analysis to two-dimensional problems: first on linear and non-linear scalar equations and finally on the Euler equations. We demonstrated that this approach yields a highly accurate estimation of the truncation error if some conditions are fulfilled. These conditions are related to the accuracy of the restriction operators, the choice of the boundary conditions, the distortion of the grids and the magnitude of the iteration error.
\end{abstract}

\section{Introduction}

In the past decade, great effort has been made to increase the accuracy of the numerical simulation of fluid flows and to decrease the computational resources required for these simulations.

Accuracy assessment is a necessary step that follows code verification and precedes model validation. Verification provides evidence that the conceptual model is solved correctly by the discrete mathematics that is embodied in the computer code. The fundamental strategy of verification is the identification, quantification, and reduction of errors in a computational model and its solution [1,2]. In this manner, numerical errors become an efficient uncertainty estimator. In addition to errors that might be introduced in the course of developing the solution algorithm, in programming or in setting up the boundary conditions, numerical solutions always include four types of systematic errors:

- Modelling error, which is defined as the difference between the actual flow and the exact solution of the mathematical model.

- Truncation error, which is defined as the difference between the discretised operator applied to the exact solution and the exact operator applied to the exact solution of the mathematical model.

- Discretisation error, which is defined as the difference between the exact solution of the mathematical model and the exact solution of the algebraic system of equations obtained by discretising the equations.

- Iteration error, which is defined as the difference between the exact solution of the algebraic system and the iterative solution of the same system.

\footnotetext{
* Corresponding author.

E-mail address: francois.fraysse@upm.es (F. Fraysse).
} 
Modelling errors are the most difficult errors to estimate. To perform this estimation, one needs data on the real flow, and in most cases, these data are not available. Therefore, modelling errors are usually estimated only for some test cases for which accurate experimental data are available or for which accurate simulations exist (i.e., direct numerical simulations). Moreover, it is important to remember that experimental data are only approximate and that the measurement and processing errors can be significant. However, these data are indispensable for the validation of models. The validation should be performed in the last step when discretisation and iteration errors have been carefully analysed.

Knowing when to stop the iteration process is crucial from the point of view of computational efficiency. As a rule of thumb, an iteration error should be at least an order of magnitude smaller than the discretisation error. Because most computational fluid dynamics solvers require the computation of the residuals, the simplest practice is to monitor their norm.

Because the discretised equations represent approximations of the differential equation, the exact solution of the latter, which is denoted by $u$, does not satisfy the difference equation. The imbalance, which is due to truncation of the Taylor series, is called a truncation error. For a grid $\Omega^{h}$ with a reference spacing $h$, the truncation error $\tau^{h}$ and the discretisation error $\epsilon^{h}$ are defined as follows:

$$
\left.\begin{array}{l}
\tau^{h}=\mathcal{L}^{h} \mathcal{I}^{h} u-\mathcal{I}^{h} \mathcal{L} u \\
\epsilon^{h}=\mathcal{I}^{h} u-u^{h}
\end{array}\right\} \Rightarrow \mathcal{L}^{h} \epsilon^{h}=\tau^{h} \text { (for linear operators) }
$$

where $\mathcal{L}$ is a symbolic operator representing the differential equation, $\mathcal{L}^{h}$ is a symbolic operator representing the algebraic equation system obtained by discretisation on grid $\Omega^{h}, \mathcal{I}^{h}$ represents a linear continuum-to-grid $\Omega^{h}$ interpolation operator (i.e., point-wise restriction) and $u^{h}$ is the exact solution of the algebraic system on the grid $\Omega^{h}$. The equation on the right side is called the discrete discretisation error transport equation (DETE, see Roy [3]).

The estimation of the numerical error provides valuable information that can be used in different applications. The truncation/discretisation errors are directly related to the mesh distribution, and thus, a careful estimation might be employed in mesh generation/mesh adaptation. These estimations might also be used to increase the accuracy of the partial differential equation (PDE) solution or in zonal modelling, as they are measures of the solution/discretisation accuracy. However, the accurate evaluation of numerical errors is not a trivial task.

A well-known strategy employed to study discretisation error is based on Richardson extrapolation [4-6]. The estimation of the discretisation error by Richardson extrapolation is based on the existence of a decomposition in the Taylor series of the solution; then, it is assumed to be a smooth solution of the partial differential equation. Recent analysis has been performed, for example, in [7-9]. The major advantage of this approach is that it is independent of the numerical scheme and thus is easily extendable to any numerical solver. However, it requires the computation of an approximated solution on at least two meshes (three if the order of accuracy of the numerical scheme is considered an unknown) with different spacing, making it unsuitable for three-dimensional industrial applications. Another family of methods employed to estimate the discretisation error is based on the solution of the DETE. In Shih [10], this approach is used to estimate the relative discretisation error (grid-induced error). However, this approach needs the solution of an auxiliary set of equations (the DETE) and an accurate model for the source term (relative truncation error). Recently, much work has been performed on the estimation of the relative discretisation error associated with functional outputs. This family of methods is called adjoint methodology, which aims to compute the linear sensitivity of an objective function with respect to a number of design variables [11,12]. Adjoint methodology permits accurate grid-induced corrections but is highly expensive, as it requires the solution of the dual problem and usually the explicit storage of an embedded grid.

The analysis of truncation error, which acts as a source term for the discretisation error through the DETE (right side of Eq. (1)), can be performed in two different manners. First, the analysis can be performed by deriving analytical expressions from Taylor series expansions, and second, the analysis can be performed by deriving a methodology to approximate the error. In the former, for example, in [13], the analysis of the exact truncation error is performed for the finite difference (FD) discretisation of the first derivative to compare its expression on the non-uniform physical domain and on the uniform transformed space. Leonard [14] investigated the differences in the truncation error when applying FD or finite volumes (FV) to the first derivative for several schemes. Jeng and Chen [15] analysed the exact truncation error arising from the FV discretisation of a steady convective equation, yielding a correction in the fluxes that improves the consistency in the presence of grid non-uniformities. Hagen [16] investigated the influence of the different terms of the truncation error with a finite element model of the linearised shallow water equations, demonstrating that their magnitude is strongly dependent on the element configuration and shape. More recently, Kallinderis [17] investigated the analytic form of the truncation error for FV discretisation of first-order derivatives, providing direct relationships between computational accuracy and elementary types of mesh distortion. However, the primary issue with these approaches is the complexity of the related expressions, particularly for multidimensional problems on arbitrary grids. The second issue is their lack of generality, as the explicit truncation error expressions differ from one scheme to another.

In contrast, the estimation of the truncation error by means of $\tau$-estimation [6] is an interesting alternative because it does not require the solution to be on a secondary grid, it only requires the computation of the residual. Furthermore, it is closely linked to the forcing term in the full approximation scheme of the multigrid approach [5], facilitating computation in a solver with a multigrid strategy. Berger [18] computed an estimation of the local truncation error using $\tau$-estimation in an adaptive FD method for the computation of a two-dimensional transonic NACA0012 Euler flow. More recently, Bernert [19] performed an extensive analysis of the accuracy of the estimation of the truncation error by $\tau$-estimation, yielding 
stringent conditions on the restriction operators for transfers from fine to coarse and coarse to fine grids. Fulton [20] proposed a different formulation, yielding less stringent conditions on the interpolation orders. However, much work has been performed using an FD method, whereas few works have been devoted to extending the application to FV solvers. Syrakos [21] successfully investigated the $\tau$-estimation for FV discretisation of the incompressible Navier-Stokes equations to increase the order of accuracy of the overall spatial scheme (procedure known as $\tau$-extrapolation [6]). However, that study did not provide extensive analyses of the accuracy of the estimations with respect to restriction operators or grid nonuniformities.

Here, we propose to extend the work of Bernert [19] and Fulton [20], who focused on uniform FD methods, to FV discretisations, both cell-centred and vertex-based, on different element types. We developed a truncation error estimator and derived all of the necessary conditions to ensure accuracy. We discuss the analysis of the conditions for an accurate estimation as follows: the order of the transfer operators acting in the truncation error estimator formula as a function of the order of the numerical scheme, the effect of the boundary conditions, the influence of distortion and the influence of the iteration error on the accuracy of the estimation. Whereas the latter were performed on one- and two-dimensional scalar equations using a cell-centred FV method, a concrete application using the vertex-based DLR TAU-Code [22] for the Euler equations is subsequently presented.

The present paper is organised as follows. First, we derive in Section 2 the mathematical formulation and the conditions to be fulfilled for an accurate estimation of the local truncation error. In Sections 3.1 and 3.2, we estimate the truncation error for one-dimensional and two-dimensional reference problems. We present the difficulties associated with this methodology as well as different solutions. Finally, in Section 4, we address more realistic configurations with Euler equations on structured and unstructured grids.

\section{Problem formulation}

Let us consider the discretisation of a partial differential equation on a grid $\Omega_{h}$ indexed by a mesh size parameter $h$ of the following form:

$$
\mathcal{L}^{h} u^{h}=f^{h}:=\mathcal{I}^{h} f
$$

where, as mentioned in the last section, $\mathcal{I}^{h}$ represents a linear continuum-to-grid $\Omega^{h}$ transfer for the specified $f$ (e.g., pointwise restriction) and $u^{h}$ represents the converged numerical solution. We recall that the corresponding local truncation error is defined as follows:

$$
\mathcal{L}^{h} \mathcal{I}^{h} u=f^{h}+\tau^{h} \rightarrow \tau^{h}=\mathcal{L}^{h} \mathcal{I}^{h} u-\mathcal{I}^{h} \mathcal{L} u
$$

In addition to the discrete equation Eq. (3) and considering a full approximation storage multigrid algorithm [6], the coarse grid equation may be written as follows:

$$
\mathcal{L}^{H} \hat{u}^{H}=\mathcal{L}^{H}\left(\widehat{\mathcal{I}}_{h}^{H} \tilde{u}^{h}\right)+\mathcal{I}_{h}^{H}\left(f^{h}-\mathcal{L}^{h} \tilde{u}^{h}\right), \quad \hat{u}^{H}=\widehat{\mathcal{I}}_{h}^{H}\left(\epsilon_{i t}^{h}+\tilde{u}^{h}\right)
$$

corresponding to the discrete equation on a coarser mesh $\Omega^{H}$, with a mesh ratio of $\rho=h / H<1$. In Eq. (4), $\tilde{u}^{h}$ is the current approximation of the solution (relaxed on the fine grid and not necessarily converged), $\epsilon_{i t}^{h}=u^{h}-\tilde{u}^{h}$ is the fine grid iteration error, for which its high frequencies must be smoothed, $\hat{I}_{h}^{H}$ represents the fine to coarse transfer operator of the solution, whereas $I_{h}^{H}$ represents the fine to coarse transfer operator of the residual. Note that these restriction operators are not necessarily identical. Similarly, introducing the relative truncation error $\tau_{h}^{H}$, Eq. (4) may be written as follows:

$$
\begin{aligned}
& \mathcal{L}^{H} \hat{u}^{H}=f^{H}+\tau_{h}^{H} \quad \text { with } \\
& \tau_{h}^{H}=\left(\mathcal{L}^{H} \widehat{\mathcal{I}}_{h}^{H} \tilde{u}^{h}-f^{H}\right)-\mathcal{I}_{h}^{H}\left(\mathcal{L}^{h} \tilde{u}^{h}-f^{h}\right)
\end{aligned}
$$

Our goal is to use $\tau_{h}^{H}$ to estimate $\tau^{H}$. If this estimation can be performed with sufficient accuracy, then one can use this local error as a mesh adaptation indicator, as an uncertainty estimator or as a means to increase the order of accuracy of the spatial scheme.

For the case in which the fine grid solution has converged, $\epsilon_{i t}^{h}=0$ then $\tilde{u}^{h}=u^{h}$, and the second right-hand side term of Eq. (6) can be neglected. Most of the analysis presented in the following sections was performed at convergence, and its extension to non-converged solutions is discussed in Section 3.1.4.

The following theorem provides the relationship between the accuracy of $\tau_{h}^{H}$ towards $\tau^{H}$ and the order of the restriction operators acting in Eq. (6).

Theorem 1 (Truncation Error Estimate). Assume that there exists $n, p, q, r, s \geqslant 1$ such that if $u \in \mathcal{C}^{n+p+q}(\Omega)$, the truncation error (3) satisfies:

- (A1) Local truncation error of order $p: \tau^{h}=h^{p} \mathcal{I}^{h} v+O\left(h^{p+q}\right)$, with $v \in \mathcal{C}^{q}(\Omega)$

- (A2) Local discretisation error of order $p: \epsilon^{h}=h^{p} \mathcal{I}^{h} w+O\left(h^{p+q}\right)$, with $w \in \mathcal{C}^{q}(\Omega)$

- (A3) Fine to coarse transfer operator of the solution of order s: $\hat{\mathcal{I}}_{h}^{H} \mathcal{I}^{h} u=\mathcal{I}^{H} u+\mathcal{O}\left(h^{s}\right)$, with $u \in \mathcal{C}^{s}(\Omega)$ 
- (A4) Fine to coarse transfer operator of the residual of order $r$ : $\mathcal{I}_{h}^{H} \mathcal{I}^{h} R=\mathcal{I}^{H} R+\mathcal{O}\left(h^{r}\right)$, with $R \in \mathcal{C}^{r}(\Omega)$

then

$$
\tau_{h}^{H}=\left(1-\rho^{p}\right) \tau^{H}+\mathcal{I}_{h}^{H} \mathcal{L}^{h} \epsilon_{i t}^{h}-\mathcal{L}^{H} \widehat{\mathcal{I}}_{h}^{H} \epsilon_{i t}^{h}+\mathcal{O}\left(h^{\min (s, p+q)}\right)
$$

for the linear case and

$$
\tau_{h}^{H}=\left(1-\rho^{p}\right) \tau^{H}+\mathcal{I}_{h}^{H}\left(\left.\frac{\partial \mathcal{L}^{h}}{\partial u^{h}}\right|_{\bar{u}^{h}} \epsilon_{i t}^{h}\right)-\left.\frac{\partial \mathcal{L}^{H}}{\partial u^{H}}\right|_{\widehat{I}_{h}^{H} \tilde{u}^{h}} \widehat{\mathcal{I}}_{h}^{H} \epsilon_{i t}^{h}+\mathcal{O}\left(\max \left(h^{\min (s, p+q, 2 p)}\right), \epsilon_{i t}^{2}\right)
$$

for the non-linear case.

In this analysis, both restriction operators $\mathcal{I}_{h}^{H} \widehat{\mathcal{I}}_{h}^{H}$, were considered linear.

The two first assumptions state that the meshes employed are sufficiently refined so that both discretisation and truncation errors decrease at the formal rate of convergence. Thus, this analysis is only valid in the asymptotic range. The main conclusion of Eq. (7) is related to the order of the restriction operator acting on the solution. Examining the exponent of the last term in Eqs. (7) and (8), it can be deduced that it is necessary to use higher order interpolation $s>p$ to transfer the solution from a fine to a coarse mesh. If $s \leqslant p$, then the truncation error estimation will be dominated by the term $\mathcal{O}\left(h^{s}\right)$, reducing the accuracy of the general results of the formula. Another interesting conclusion of Eqs. (7) and (8) is that the formulas are independent of the order $r$ of the restriction operator for the residual. Next, we derived a specific condition for this restriction operator to estimate the truncation error in the case of a non-converged solution.

Proof. We made a distinction between linear and non-linear differential operators, both for converged and non-converged solutions, as follows.

- Linear case

- Converged solution

From (A2) we get:

$$
\epsilon^{H}-\widehat{\mathcal{I}}_{h}^{H} \epsilon^{h}=\left(1-\rho^{p}\right) \epsilon^{H}+\mathcal{O}\left(h^{p+\min (q, s)}\right)
$$

For the converged case, $\tilde{u}^{h}=u^{h}, \epsilon_{i t}^{h}=0$. Then, we decomposed $u^{h}$ such that $u^{h}=\mathcal{I}^{h} u-\epsilon^{h}$, and, using the fact that $L^{H}$ is linear, Eq. (6) becomes

$$
\tau_{h}^{H}=\mathcal{L}^{H} \widehat{\mathcal{I}}_{h}^{H}\left(\mathcal{I}^{h} u-\epsilon^{h}\right)-f^{H}=\mathcal{L}^{H} \widehat{\mathcal{I}}_{h}^{H} \mathcal{T}^{h} u-f^{H}-\mathcal{L}^{H} \widehat{\mathcal{I}}_{h}^{H} \epsilon^{h}
$$

Using assumption (A3) and Eqs. (1) and (10) becomes

$$
\tau_{h}^{H}=\tau^{H}-\mathcal{L}^{H} \hat{\mathcal{I}}_{h}^{H} \epsilon^{h}+\mathcal{O}\left(h^{s}\right)
$$

Now, by the use of Eqs. (9) and (11) becomes

$$
\tau_{h}^{H}=\tau^{H}-\rho^{p} \mathcal{L}^{H} \epsilon^{H}+\mathcal{O}\left(h^{\min (s, p+q)}\right)
$$

Finally, using the relation $\mathcal{L}^{H} \epsilon^{H}=\tau^{H}$, Eq. (12) becomes

$$
\tau_{h}^{H}=\left(1-\rho^{p}\right) \tau^{H}+\mathcal{O}\left(h^{\min (s, p+q))}\right)
$$

- Non-converged solution

In the non-converged case, let us decompose the approximate solution $\tilde{u}^{h}$ such that $\tilde{u}^{h}=\mathcal{I}^{h} u-\epsilon^{h}-\epsilon_{i t}^{h}$. The first term $T_{1}$ of Eq. (6) becomes

$$
T_{1}=\mathcal{L}^{H} \widehat{\mathcal{I}}_{h}^{H}\left(\mathcal{I}^{h} u-\epsilon^{h}-\epsilon_{i t}^{h}\right)-f^{H}
$$

Similar to the converged case, and using the fact that $\mathcal{L}^{H}$ is linear, we obtain

$$
T_{1}=\mathcal{L}^{H} \hat{\mathcal{I}}_{h}^{H}\left(\mathcal{I}^{h} u-\epsilon^{h}-\epsilon_{i t}^{h}\right)-f^{H}=\left(1-\rho^{p}\right) \tau^{H}-\mathcal{L}^{H} \hat{\mathcal{I}}_{h}^{H} \epsilon_{i t}^{h}+\mathcal{O}\left(h^{\min (s, p+q))}\right)
$$

The second term $T_{2}$ of Eq. (6), using Eq. (3), becomes

$$
T_{2}=-\mathcal{I}_{h}^{H}\left(\mathcal{L}^{h}\left(\mathcal{I}^{h} u-\epsilon^{h}-\epsilon_{i t}^{h}\right)-f^{h}\right)=-\mathcal{I}_{h}^{H}\left(\tau^{h}-\mathcal{L}^{h} \epsilon^{h}-\mathcal{L}^{h} \epsilon_{i t}^{h}\right)
$$

Now, using the relation $\mathcal{L}^{h} \epsilon^{h}=\tau^{h}$, we finally obtain

$$
T_{2}=\mathcal{I}_{h}^{H} \mathcal{L}^{h} \epsilon_{i t}^{h}
$$

Joining the terms $T_{1}+T_{2}=\tau_{h}^{H}$, we obtain

$$
\tau_{h}^{H}=\left(1-\rho^{p}\right) \tau^{H}+\mathcal{I}_{h}^{H} \mathcal{L}^{h} \epsilon_{i t}^{h}-\mathcal{L}^{H} \widehat{\mathcal{I}}_{h}^{H} \epsilon_{i t}^{h}+\mathcal{O}\left(h^{\min (s, p+q)}\right)
$$


The second and third terms on the right-hand side of this equation are the difference between the fine to coarse interpolation of the differential operator applied over the fine grid iteration error $\left(\mathcal{I}_{h}^{H} \mathcal{L}^{h} \epsilon_{i t}^{h}\right)$ and the differential operator on the coarse mesh applied over the fine to coarse interpolation of the fine grid iteration error $\left(\mathcal{L}^{H} \hat{\mathcal{I}}_{h}^{H} \epsilon_{i t}^{h}\right)$, respectively. When $\epsilon_{i t}^{h}$ is sufficiently small, these two terms can be neglected. However, in the general case when the solution is not converged, the iteration error can be of order unity, and thus, these terms disappear only if the following relation holds:

$$
\mathcal{I}_{h}^{H} \mathcal{L}^{h}=\mathcal{L}^{H} \widehat{\mathcal{I}}_{h}^{H}
$$

Normally, the operator $\mathcal{L}^{H}$ is obtained by discretisation coarse grid approximation (dca) [23], which means that the coarse grid operator is the same as the fine grid operator, but it is applied over the coarse mesh. In this case, for a given $\widehat{\mathcal{I}}_{h}^{H}$, it is possible to eliminate the two last terms of Eq. (7) if the restriction operator applied to the residual $\mathcal{I}_{h}^{H}$ satisfies the following equation:

$$
\mathcal{I}_{h}^{H}=\mathcal{L}_{\text {dca }}^{H} \widehat{\mathcal{I}}_{h}^{H}\left(\mathcal{L}^{h}\right)^{-1}
$$

The other method of eliminating the two last terms of Eq. (7) is to define the coarse grid differential operator for both given restriction operators in such a way that it fulfils Eq. (16). This procedure is known as Galerkin coarse grid approximation (see [23]).

However, in the general case, the discretisation coarse grid approximation method is employed. Then, if we want to apply this methodology to any type of "existing" solvers, we do not consider the implementation of a new coarse grid operator. Moreover, if no attention is paid to the choice of $\mathcal{L}_{h}^{H}$, then we can easily examine the following approximation instead:

$$
\mathcal{I}_{h}^{H} \mathcal{L}^{h} \epsilon_{i t}^{h}=\mathcal{L}^{H} \hat{\mathcal{I}}_{h}^{H} \epsilon_{i t}^{h}+\operatorname{ch}^{l} \widehat{\mathcal{I}}_{h}^{H}\left(\epsilon_{i t}^{h}\right)^{(m)}+\mathcal{O}\left(h^{l+1}\right)
$$

with $c$ a real constant and $l, m$ positive integers depending on the operator used. We present a detailed analysis of the effect of different restriction operators on the estimation of the truncation error in the transient phase in Section 3.1.4.

- Non-linear case

- Converged solution

For the converged case, $\tilde{u}^{h}=u^{h}, \epsilon_{i t}^{h}=0$ and Eq. (6) are reduced to the following:

$$
\tau_{h}^{H}=\mathcal{L}^{H} \hat{\mathcal{I}}_{h}^{H} u^{h}-f^{H}
$$

Now, the relationship $\tau^{h}=\mathcal{L}^{h} \epsilon^{h}$ is no longer valid. Instead, the following relationship holds:

$$
\tau^{h}=\mathcal{L}^{h}\left(u^{h}+\epsilon^{h}\right)-f^{h} \Rightarrow \tau^{h}=\left.\frac{\partial \mathcal{L}^{h}}{\partial u^{h}}\right|_{u^{h}} \epsilon^{h}+\mathcal{O}\left(h^{2 p}\right),
$$

where $\frac{\partial \mathcal{L}^{h}}{\partial u^{h}}$ is the local Jacobian of $\mathcal{L}^{h}$ obtained on grid $h$ (respectively, $H$ ). Similar to the linear case, if we decompose $u^{h}$ such that $u^{h}=u^{\partial u^{h}} u-\epsilon^{h}$, we obtain

$$
\tau_{h}^{H}=\mathcal{L}^{H} \hat{\mathcal{I}}_{h}^{H}\left(\mathcal{I}^{h} u-\epsilon^{h}\right)-f^{H}
$$

Furthermore, by linearising $\mathcal{L}^{H} \hat{\mathcal{I}}_{h}^{H}\left(\mathcal{I}^{h} u-\epsilon^{h}\right)$ and using (A3), we obtain

$$
\tau_{h}^{H}=\tau^{H}-\left.\frac{\partial \mathcal{L}^{H}}{\partial u^{H}}\right|_{I^{H} u} \hat{\mathcal{I}}_{h}^{H} \epsilon^{h}+\mathcal{O}\left(h^{\min (s, 2 p)}\right)=\tau^{H}-\left.\frac{\partial \mathcal{L}^{H}}{\partial u^{H}}\right|_{u^{H}} \rho^{p} \epsilon^{H}+\mathcal{O}\left(h^{\min (s, p+q, 2 p)}\right)
$$

Finally, Eq. (20) becomes

$$
\tau_{h}^{H}=\left(1-\rho^{p}\right) \tau^{H}+\mathcal{O}\left(h^{\min (s, p+q, 2 p)}\right)
$$

- Non-converged solution

For the non-converged case, by proceeding in an equivalent manner to the linear analysis, assuming that $\epsilon_{i t}^{h} \ll \mathcal{I}^{h} u$ and using Eq. (19), we finally obtain

$$
\tau_{h}^{H}=\left(1-\rho^{p}\right) \tau^{H}+\mathcal{I}_{h}^{H}\left(\left.\frac{\partial \mathcal{L}^{h}}{\partial u^{h}}\right|_{\bar{u}^{h}} \epsilon_{i t}^{h}\right)-\left.\frac{\partial \mathcal{L}^{H}}{\partial u^{H}}\right|_{\widehat{I}_{h}^{H} \hat{h}^{h}} \widehat{\mathcal{I}}_{h}^{H} \epsilon_{i t}^{h}+\mathcal{O}\left(\max \left(h^{\min (s, p+q, 2 p)}\right), \epsilon_{i t}^{2}\right)
$$

Now, in contrast to the linear case, deriving a relationship with the restriction operators that eliminates the remaining terms does not necessarily ensure that the estimation will be accurate before any relaxation is performed because of the magnitude of the iteration error in the last term of the present equation. To derive this equation, linearisations must be performed assuming that $\epsilon_{i t}^{h} \ll \mathcal{I}^{h} u$.

However, in general, the Jacobian of the nonlinear operator is not necessarily smooth, and the iteration error for a nonsmooth solution can be locally large. In any case, the $\tau$-estimation formula is still valid, but now it is more problem dependent. According to our numerical experiments, for smooth solutions, this formula is not valid at the first iteration, but still provides accurate estimations of the truncation error far before the solution has converged.

In any case, further analysis of the structure of the Jacobian and its relationship with the restriction operators should be performed in this context. 
To validate Eqs. (7) and (8), for the linear and non-linear cases, respectively, we employed a set of partial differential equations with known analytic solutions in the following sections. First, we emphasised the analysis on one-dimensional equations, and then we investigated the extension to two-dimensional scalar and vectorial problems.

\section{Detailed analysis on reference problems}

Here, we studied the effects on the truncation error estimation of the order of the restriction operators, the boundary conditions, the non-uniform meshes and the iteration error for non-converged solutions, both for FD and for FV methods. In the following section, the truncation error estimation is compared with the exact truncation error. The latter, with an analytic solution in hand, is computed using Eq. (1).

In the next section, the analytic solutions follow the method of manufactured solution (Roache [2]). The concept behind this method is to build a solution and modify the governing equations by inserting the source term that makes the manufactured solution verify the problem. Trigonometric functions were employed because no derivative can be eliminated, which is of importance to analyse the order of accuracy.

The analysis of the estimated accuracy of the truncation error is measured on successive meshes of different spacing by monitoring the $L_{\infty}$ norm of the error in the truncation error estimation (e.g. $\left\|\tau^{H}-\frac{1}{1-\rho^{p}} \tau_{h}^{H}\right\|_{\infty}$, with $\rho=h / H$ and $p$ the formal order of the numerical scheme). The requirements for a systematic mesh refinement can be found in Oberkampf and Roy [1], who state that the successively refined grids should satisfy a uniform refinement and a consistent refinement. The most important conclusion is that the grid quality has to remain constant or improve through refinement. Clearly, the asymptotic behaviour is dependent on the base grid, but above all, it is dependent on the refinement procedure. In the following sections, we consider uniform (in Section 3.1.1), smooth non-uniform and highly distorted grids (in Section 3.1.3) for which a different systematic mesh refinement procedure is proposed depending on the features analysed.

\subsection{One-dimensional test cases}

First, to illustrate the accuracy of the local truncation error estimate based on the formulation of Eq. (6), we considered the one-dimensional diffusion (linear) and diffusion-convection (non-linear, Burger's equation) equations with known exact solutions. In these cases, we can compute the exact truncation error by injecting the exact solution into Eq. (1).

The one-dimensional equations considered read as follows:

$$
-u^{\prime \prime}=f_{1}+\text { b.c. }, \quad-u^{\prime \prime}+u u^{\prime}=f_{2}+\text { b.c }
$$

where b.c represents the boundary conditions. Furthermore, we considered the following test functions:

$$
\left\{\begin{array} { l } 
{ f _ { 1 } ( x ) = 1 6 \operatorname { c o s } ( 4 x ) } \\
{ u ( 0 ) = u _ { e x } ( 0 ) , \quad u ( 1 ) = u _ { e x } ( 1 ) }
\end{array} \quad \left\{\begin{array}{l}
f_{2}(x)=16 \cos (4 x)-4 \sin (4 x) \cos (4 x) \\
u(0)=u_{e x}(0), \quad u(1)=u_{e x}(1)
\end{array}\right.\right.
$$

These problems have the following exact solution:

$$
u_{e x}(x)=\cos (4 x) \text {. }
$$

Eqs. (23) and (24) are solved using second-order spatial schemes. An FD method associated with a second-order accurate central scheme for the computation of the second derivative was considered. Concerning the FV approach, a second-order central scheme for the computation of the fluxes was used. The non-linear term was computed using a central scheme for the computation of the first derivative.

The steady-state solution was reached using a Runge-Kutta relaxation scheme and a multigrid strategy.

We focused the analysis on the different aspects that influence the estimation of the truncation error. First, on the converged solutions, we studied the order of the restriction operators for the solution. Then, we performed a deeper examination of the boundaries of the domain, particularly when applying the analytic solution or an analytic flux. We continued with the analysis of the accuracy of the estimator in the presence of non-uniform grids. Finally, we concluded the one-dimensional analysis with an estimation of the truncation error when the solution has not converged.

\subsubsection{Influence of the order of the restriction operator for the solution $\left(\hat{\mathcal{I}}_{h}^{H}\right)$}

Here, we restricted the analysis to converged solutions on uniform grids for both FD and FV methods. These two discretisations give the following truncation error expressions for a uniform grid $\Omega_{h}$ :

$$
\begin{aligned}
& \mathcal{L}^{h} \mathcal{I}^{h} u=\frac{-u_{i+1}+2 u_{i}-u_{i-1}}{h^{2}} \Rightarrow \tau^{h}\left(x_{i}\right)=-\frac{h^{2}}{12} \frac{\partial^{4} u_{i}}{\partial x^{4}}+\mathcal{O}\left(h^{4}\right) \\
& \mathcal{L}^{h} \mathcal{I}^{h} u=\frac{-u_{i+1}+2 u_{i}-u_{i-1}}{h^{2}}+u_{i} \frac{u_{i+1}-u_{i-1}}{2 h} \Rightarrow \tau^{h}\left(x_{i}\right)=-\frac{h^{2}}{12} \frac{\partial^{4} u_{i}}{\partial x^{4}}+\frac{h^{2}}{6} u_{i} \frac{\partial^{3} u_{i}}{\partial x^{3}}+\mathcal{O}\left(h^{4}\right)
\end{aligned}
$$

Note that in the specific case of uniform grids, the FD and FV methods yield the same discretisation. From Eqs. (25) and (26), we observe that the dominating term of the truncation error is of order $2(p=2)$ and the following term of order $4(q=2)$ for both linear and non-linear problems. 
Here, we analysed the magnitude of the error in the estimation as the mesh spacing is reduced. In this aim, a set of successively refined uniform grids was built from $\max =9$ to $i \max =129$. At each grid level, a coarse grid was extracted by removing one point every two points to compute the truncation error estimate from Eq. (6) so that in the following $\rho=h / H=1 / 2$, where $h$ and $H$ are the spacing of a fine grid and the first coarse grid, respectively.

The estimation of the local truncation error was performed using several restriction operators to interpolate the solution from the fine grid level to the first coarse level.

The classic multigrid approach used in conjunction with the FD method naturally allows an injective operator to restrict the solution from the fine grid level to the coarse grid level. In the case of a cell-centred FV and in the common case where the coarse grid is obtained by removing one point every two points, an exact restriction such as injection cannot be performed, as the barycentre of a coarse grid cell does not coincide with any barycentre of a fine grid cell. As a result, in the following section, injection was used for the FD method, whereas the second- (the so-called full weighting), third- and fourth-order restriction operators for the solution were tested for the FV method.

The $L_{\infty}$ norm of the discretisation error, the exact truncation error and the error in the estimation $\left\|\tau^{H}-4 / 3 \tau_{h}^{H}\right\|_{\infty}$ were computed and reported in Figs. 1 and 2. The numerical experiments follow the conclusions of the analysis of Section 2 for both the linear and the non-linear problems.

When the FD formulation is used in conjunction with injection to restrict the solution, Eq. (7) (linear) and Eq. (8) (nonlinear) hold with $p=q=2$ and $s=\infty$; then, we expect the error in the estimation $\left\|\tau^{H}-4 / 3 \tau_{h}^{H}\right\|_{\infty}$ to be of the order of $p+q=4$. When the cell-centred FV method is used, Eq. (7) holds with $p=q=2$ and $s=2,3,4$ for the full weighting and the third-and fourth-order restrictions, respectively. Then, we expect the error in the estimation $\left\|\tau^{H}-4 / 3 \tau_{h}^{H}\right\|_{\infty}$ to be of order of two, three and four, respectively. When the second-order (full-weighting, $s=2$ ) operator is used to restrict the solution of the FV method, the estimation of the local truncation error is no longer accurate in an asymptotic sense because the exact truncation error for this specific problem is of the order of two.

\subsubsection{Boundary condition analysis}

The previous analysis has been performed by omitting a boundary strip of length $1 / 8$ of the domain. In fact, in the regions close to the boundaries, the $L_{\infty}$ norm of the error in the estimation $\left\|\tau^{H}-4 / 3 \tau_{h}^{H}\right\|_{\infty}$ drops to a second-order magnitude. This problem is due to the boundary condition that is imposed on the coarse mesh. Whereas the interior nodes are restricted from the fine mesh using, for example, three fine grid points for third-order interpolation, the use of the same stencil for the coarse boundary points is impossible without using additional ghost cells, which is undesirable because it is code intrusive. Thus, to restrict the fine grid solution onto the coarse grid ghost cell, a third-order extrapolation must be employed. Because of the accuracy issues related to high-order extrapolations, we wished to avoid this procedure. When an analytical solution is known, the natural method would be to impose the Dirichlet condition as well at the coarse grid boundary points; however, as indicated in the following section, this strategy does not permit accurate $\tau$-estimation at the cells adjacent to the boundaries.

Here, we analysed this phenomenon using the one-dimensional FV method on the linear problem, a third-order operator $(s=3)$ to restrict the solution and a first-order operator $(r=1)$ to restrict the residual for uniform grids. Therefore, developing the Taylor series around $\tilde{u}_{i}$, and following the notation of Fig. 3, we obtain
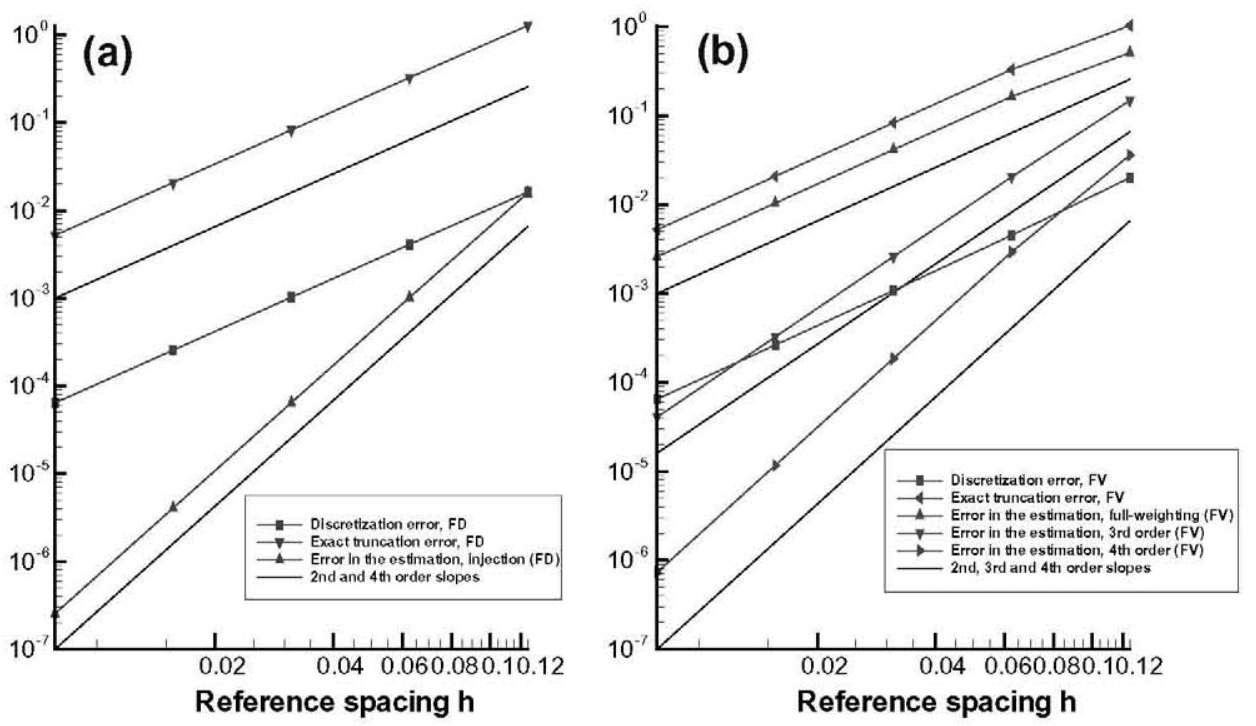

Fig. 1. $\|\cdot\|_{L_{\infty}}$ of the error in the truncation error estimate for the $1 \mathrm{D}$ linear problem on (a): uniform grids using finite difference method, (b): uniform grids using finite volume method. 

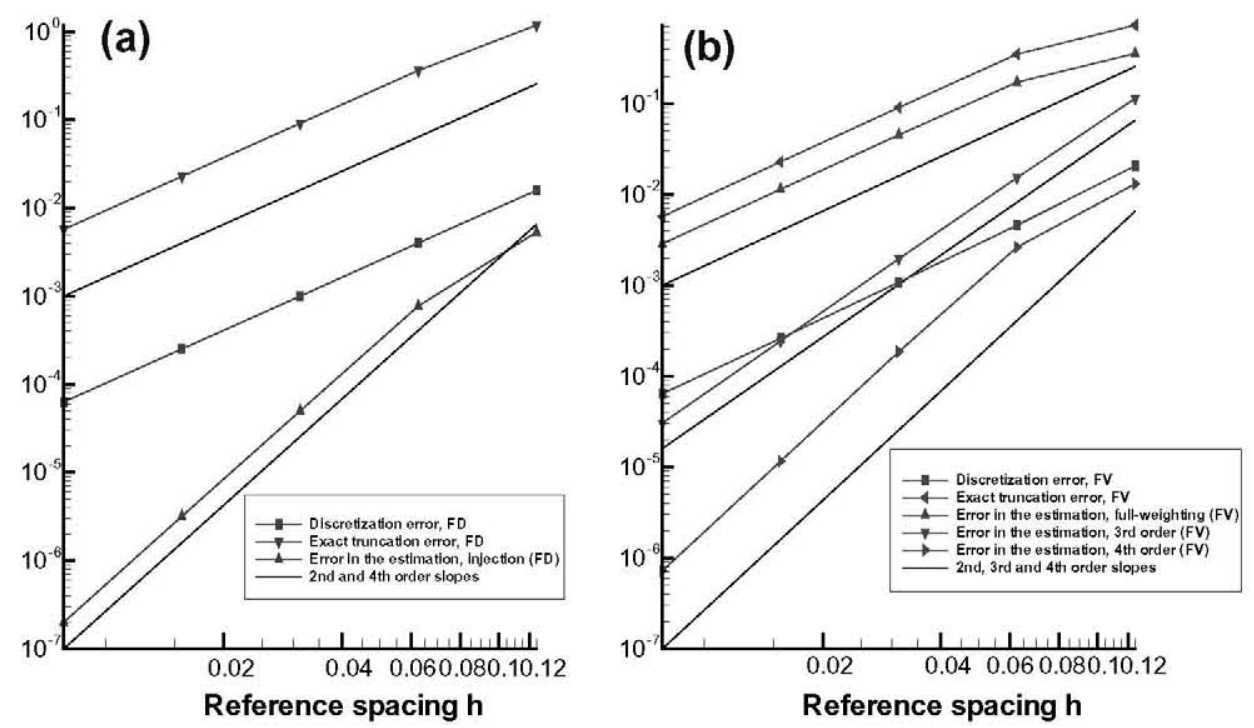

Fig. 2. $\|\cdot\|_{L_{\infty}}$ of the error in the truncation error estimate for the 1D non-linear problem on (a): uniform grids using finite difference method, (b): uniform grids using finite volume method.

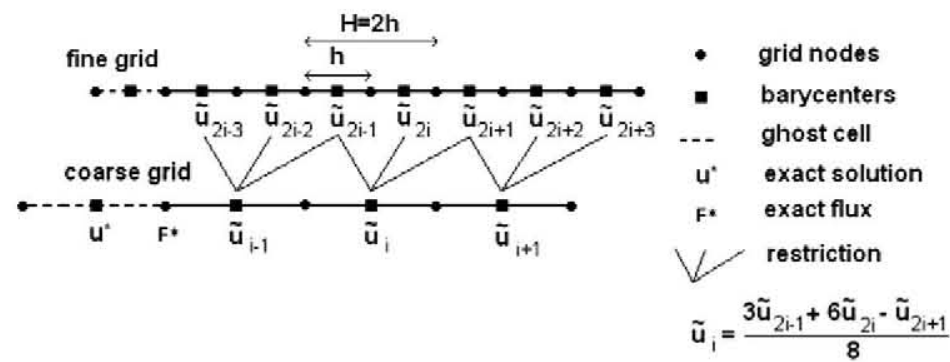

Fig. 3. Fine and coarse grid notations.

$$
\tilde{u}_{2 i+j}=\tilde{u}_{i}+\sum_{p=1}^{6} \alpha_{j p} h^{p} \frac{\partial^{p} \tilde{u}_{i}}{\partial x^{p}}
$$

Using the coefficients $\alpha_{j p}$ explicitly stated in Table 1, we obtain

$$
\frac{4}{3}\left(\tau_{h}^{H}\right)_{i}=\frac{-3 \tilde{u}_{2 i-3}-6 \tilde{u}_{2 i-2}+39 \tilde{u}_{2 i-1}-52 \tilde{u}_{2 i}+27 \tilde{u}_{2 i+1}-6 \tilde{u}_{2 i+2}+\tilde{u}_{2 i+3}}{32 h^{2}}-f_{i}+f_{2 i}=-\frac{H^{2}}{12} \frac{\partial^{4} \tilde{u}_{i}}{\partial x^{4}}+\frac{5 H^{3}}{288} \frac{\partial^{5} \tilde{u}_{i}}{\partial x^{5}}=\tau^{H}+\mathcal{O}\left(H^{3}\right)
$$

which is valid for the interior nodes and fulfils Eq. (7), by inserting the Taylor developments of Eq. (27) into Eq. (6), in the general case (considering $\rho=1 / 2$ ), with the operator $\mathcal{L}^{h}$ defined in Eq. (25).

However, at the cells adjacent to the boundary, if we denote the Dirichlet condition imposed in the ghost cell of the coarse mesh $\Omega_{H}$ by $u^{*}$ (see Fig. 3), we obtain

$$
\left(\tau_{h}^{H}\right)_{i-1}=\frac{-26 \tilde{u}_{2 i-3}+76 \tilde{u}_{2 i-2}-36 \tilde{u}_{2 i-1}-6 \tilde{u}_{2 i}+\tilde{u}_{2 i+1}-u^{*}}{32 h^{2}}-f_{i-1}+f_{2 i-2}
$$

To accurately estimate the truncation error at the cells adjacent to the boundary, we then need the following relation for $u^{*}$ to hold:

$$
\begin{aligned}
& \left(\tau_{h}^{H}\right)_{i-1}=-\frac{H^{2}}{12} \frac{\partial^{4} \tilde{u}_{i-1}}{\partial x^{4}}+\mathcal{O}\left(H^{4}\right) \Longleftrightarrow \\
& u^{*}=11 \tilde{u}_{i-1}-\frac{29 H}{4} \frac{\partial \tilde{u}_{i-1}}{\partial x}-\frac{375 H^{2}}{32} \frac{\partial^{2} \tilde{u}_{i-1}}{\partial x^{2}}-\frac{4349 H^{3}}{384} \frac{\partial^{3} \tilde{u}_{i-1}}{\partial x^{3}}-\frac{1655 H^{4}}{6144} \frac{\partial^{4} \tilde{u}_{i-1}}{\partial x^{4}}-\frac{4383 H^{5}}{40960} \frac{\partial^{5} \tilde{u}_{i-1}}{\partial x^{5}}
\end{aligned}
$$


Table 1

Taylors series coefficients.

\begin{tabular}{|c|c|c|c|c|c|c|}
\hline \multirow[t]{2}{*}{$j$} & \multicolumn{6}{|l|}{$p$} \\
\hline & 1 & 2 & 3 & 4 & 5 & 6 \\
\hline-3 & $-5 / 2$ & $25 / 8$ & $-125 / 48$ & $625 / 384$ & $-3125 / 3840$ & $15625 / 46080$ \\
\hline-2 & $-3 / 2$ & $9 / 8$ & $-27 / 48$ & $81 / 384$ & $-243 / 3840$ & $729 / 46080$ \\
\hline-1 & $-1 / 2$ & $1 / 8$ & $-1 / 48$ & $1 / 384$ & $-1 / 3840$ & $1 / 46080$ \\
\hline 0 & $1 / 2$ & $1 / 8$ & $1 / 48$ & $1 / 384$ & $1 / 3840$ & $1 / 46080$ \\
\hline 1 & $3 / 2$ & $9 / 8$ & $27 / 48$ & $81 / 384$ & $243 / 3840$ & $729 / 46080$ \\
\hline 2 & $5 / 2$ & $25 / 8$ & $125 / 48$ & $625 / 384$ & $3125 / 3840$ & $15625 / 46080$ \\
\hline 3 & $7 / 2$ & $49 / 8$ & $343 / 48$ & $2401 / 384$ & $16807 / 3840$ & $117649 / 46080$ \\
\hline
\end{tabular}

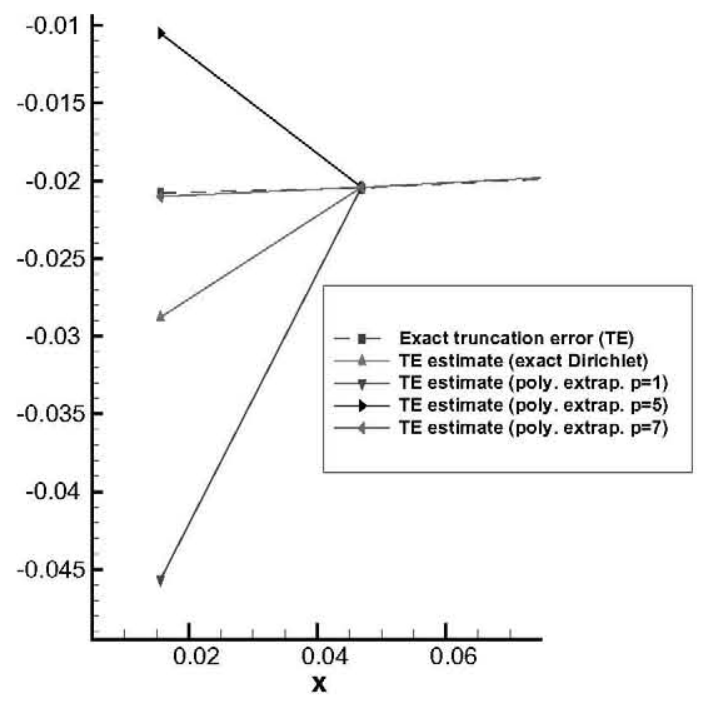

Fig. 4. Extrapolation of the coarse boundary condition from the interior nodes.

Conversely, if we impose a Neumann boundary condition $F^{*}$ on $\partial \Omega_{H}$, we obtain

$$
\left(\tau_{h}^{H}\right)_{i}=\frac{-29 \tilde{u}_{2 i-1}+70 \tilde{u}_{2 i}-36 \tilde{u}_{2 i+1}-6 \tilde{u}_{2 i+2}+\tilde{u}_{2 i+3}}{32 h^{2}}-\frac{F^{*}}{2 h}-f_{i}+f_{2 i}
$$

Then, we need the flux at the boundary to satisfy the following:

$$
\begin{aligned}
& \left(\tau_{h}^{H}\right)_{i}=-\frac{H^{2}}{12} \frac{\partial^{4} \tilde{u}_{i}}{\partial x^{4}}+\mathcal{O}\left(H^{4}\right) \Longleftrightarrow \\
& F^{*}=-\frac{\partial \tilde{u}_{i}}{\partial x}-\frac{3 H}{2} \frac{\partial^{2} \tilde{u}_{i}}{\partial x^{2}}-\frac{17 H^{2}}{2} \frac{\partial^{3} \tilde{u}_{i}}{\partial x^{3}}-\frac{113 H^{3}}{128} \frac{\partial^{4} \tilde{u}_{i}}{\partial x^{4}}-\frac{411 H^{4}}{30720} \frac{\partial^{5} \tilde{u}_{i}}{\partial x^{5}}
\end{aligned}
$$

This means that if we impose the exact solution $u^{*}$ in the ghost cell or the exact flux $F^{*}$ at the boundary of the coarse mesh, this construct adds a source of error in the estimation of the truncation error using Eq. (6). To overcome this problem practically, we can extrapolate the boundary condition of the coarse mesh from the grid nodes located in the interior of the domain. To illustrate this problem, in Fig. 4, the estimation of the truncation error is reported after imposing the Dirichlet boundary condition and extrapolating the value of the ghost cell from the interior nodes. It is demonstrated that the Dirichlet boundary condition causes a source of error in the cells adjacent to the boundary; this error decreases when the extrapolation order increases. However, a large stencil is required to obtain an accurate truncation error estimation. This procedure is easily realisable on one-dimensional structured grids. Higher-order extrapolation in multi-dimensional hybrid solvers is not an easy task; as a result, in practical problems, it would be simpler to extrapolate the truncation error estimate. This problem can be of importance for Euler or Navier-Stokes equations, if, for example, the isothermal wall bounded boundary condition is imposed.

\subsubsection{Non-uniform grids}

In this section, we focused the analysis of the accuracy of the truncation error estimate on non-uniform grids. The analysis is restricted to the converged solution of the linear problem of Eq. (23). 


$$
\begin{aligned}
\mathcal{L}^{h} I^{h} u= & \frac{-2 h_{i-1} u_{i+1}+2\left(h_{i}+h_{i-1}\right) u_{i}-2 h_{i} u_{i-1}}{h_{i} h_{i-1}\left(h_{i}+h_{i-1}\right)} \\
\Rightarrow \tau^{h}\left(x_{i}\right)= & -\frac{h_{i}-h_{i-1}}{3} \frac{\partial^{3} u_{i}}{\partial x^{3}} \\
& -\frac{h_{i}^{2}-h_{i} h_{i-1}+h_{i-1}^{2}}{12} \frac{\partial^{4} u_{i}}{\partial x^{4}}+O\left(h^{3}\right)
\end{aligned}
$$

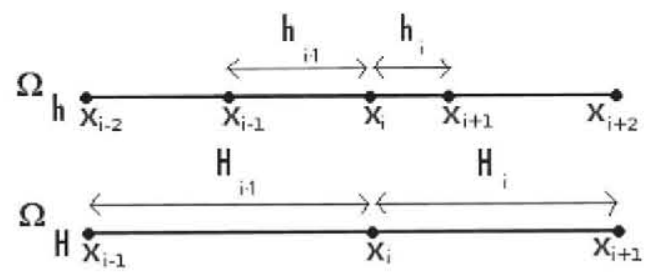

Fig. 5. 1D Finite difference method.

The FD strategy employs a central discretisation, whereas the cell-centred FV method uses a central scheme to compute the first derivatives at the interfaces of the cells. For the linear case, these spatial discretisations yield the truncation error formulas of Eq. (28) for the FD method and Eq. (29) for the FV method with the notation explained in Figs. 5 and 6 , respectively.

$$
\begin{aligned}
\mathcal{L}^{h} \mathcal{I}^{h} u= & \frac{-2 h_{i-1} u_{i+1}+2\left(h_{i}+h_{i-1}\right) u_{i}-2 h_{i} u_{i-1}}{h_{i} h_{i-1}\left(h_{i}+h_{i-1}\right)} \\
\Rightarrow \tau^{h}\left(x_{i}\right)= & -\frac{h_{i}-h_{i-1}}{3} \frac{\partial^{3} u_{i}}{\partial x^{3}} \\
& -\frac{h_{i}^{2}-h_{i} h_{i-1}+h_{i-1}^{2}}{12} \frac{\partial^{4} u_{i}}{\partial x^{4}}+\mathcal{O}\left(h^{3}\right) \\
\mathcal{L}^{h} \mathcal{I}^{h} u= & \frac{1}{x_{i+1}-x_{i}}\left[\frac{u_{i+1}-u_{i}}{h_{i}}-\frac{u_{i}-u_{i-1}}{h_{i-1}}\right] \quad(\mathrm{FV}) \\
\Rightarrow \tau^{h}\left(x_{i}^{c}\right)= & {\left[\frac{\partial^{2} u_{i}}{\partial x^{2}}-\frac{h_{i}+h_{i-1}}{2\left(x_{i+1}-x_{i}\right)} \frac{\partial^{2} u_{i}}{\partial x^{2}}\right] } \\
& -\frac{h_{i}^{2}-h_{i-1}^{2}}{6\left(x_{i+1}-x_{i}\right)} \frac{\partial^{3} u_{i}}{\partial x^{3}} \\
& -\frac{h_{i}^{3}+h_{i-1}^{3}}{24\left(x_{i+1}-x_{i}\right)} \frac{\partial^{4} u_{i}}{\partial x^{4}}+\mathcal{O}\left(h^{3}\right)
\end{aligned}
$$

It can be observed in Eqs. (28) and (29) that the truncation error has two different main contributions. Extra terms appear because of grid non-uniformity; note that Eq. (25) is recovered in the case of uniform grids. If the grid is highly distorted, the truncation error is dominated by these terms. For smooth meshes, e.g., $h_{i}-h_{i-1} \sim h_{i}^{2}$ or higher, the truncation error attains second-order precision, which is the known performance of central schemes on smooth meshes. At this point, it is important to stress that, for extremely distorted meshes, the magnitude of the truncation error becomes first order or order unity. 


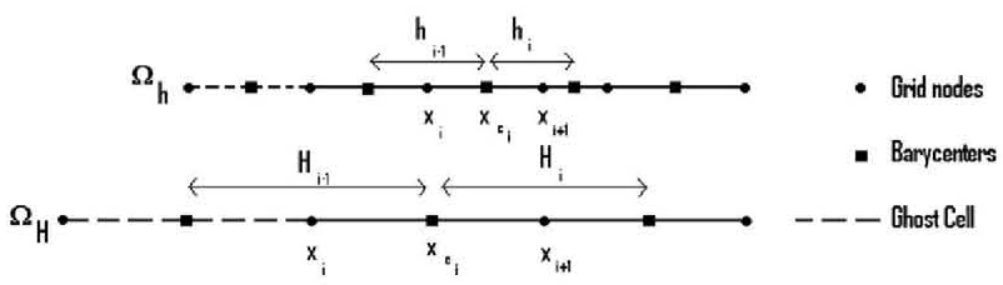

Fig. 6. 1D Finite volume method.

It is also worth mentioning that Eq. (28) (FD) and Eq. (29) (FV) differ when the grid is non-uniform. In the case of highly distorted meshes, the FV method yields an inconsistency because of the use of a non-weighted central scheme for the computation of the fluxes at the interfaces of the grid cells. This problem has been previously reported by other authors, such as Jeng [15].

In the following section, we considered two distinct grid distributions. First, we considered a smooth, non-uniform grid distribution that maintains a second-order truncation error for both the FD and the FV methods. Second, we investigated the accuracy of the truncation error estimate for both discretisation approaches on highly distorted grids. Two different systematic mesh refinement strategies were employed to analyse the accuracy of the truncation error estimate. For the smooth nonuniform case, the set of grids was built in such a way that the mesh quality increases with the refinement. Concerning the highly distorted grid, a set of grids was built in such a way that the quality of the grid was maintained throughout the refinement. The reasons for using different systematic mesh refinement strategies are related to the specific feature we analysed, and they are explained in the following section.

\section{Smooth non-uniform grids}

The earlier equations of Eq. (28) (FD) and Eq. (29) (FV) indicated the presence of additional terms related to the non-uniformity of the grid. To maintain a second-order truncation error, we propose to use the following grid distribution:

$$
\left\{\begin{array}{l}
\eta_{i}=\frac{i-1}{\operatorname{imax}-1} \quad i=1, \ldots, \text { imax } \\
B=\frac{1}{2 \alpha} \ln \left(\frac{1+\left(e^{\alpha}-1\right) x_{0}}{1-\left(1-e^{-\alpha}\right) x_{0}}\right) \\
x_{i}=x_{0}\left(1+\frac{\sinh \left(\alpha\left(\eta_{i}-B\right)\right)}{\sinh (\alpha B)}\right)
\end{array}\right.
$$

where imax is the overall number of nodes in the $x$-direction, $\alpha$ controls the stretching ( $\alpha=6$ is used in this work) and $x_{0}$ is the location in the domain where the grid lines are to be clustered, here set in the middle of the domain $x_{0}=0.5$. This distribution yields smooth grids, as the difference of two successive intervals is of the order of 2, ensuring that the non-uniform and inconsistency terms of Eqs. (28) and (29) decrease at least at a second-order rate when the grid is refined, as indicated in Fig. 7.

We analysed, as in Section 3.1.1, the magnitude of the error in the estimation $\left\|\tau^{H}-4 / 3 \tau_{h}^{H}\right\|_{\infty}$ as the mesh spacing is refined using different restriction operators for the solution. An initial imax $=129$ grid was computed using Eq. (30); then, four

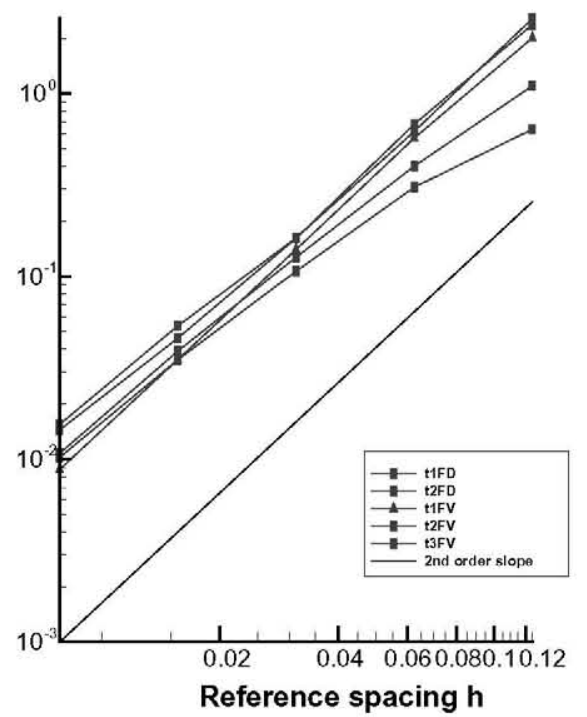

Fig. 7. $\|\cdot\|_{L_{\infty}}$ of the non-uniform terms in the truncation error expressions (Eqs. (28) and (29)) using grid distribution from Eq. (30). 

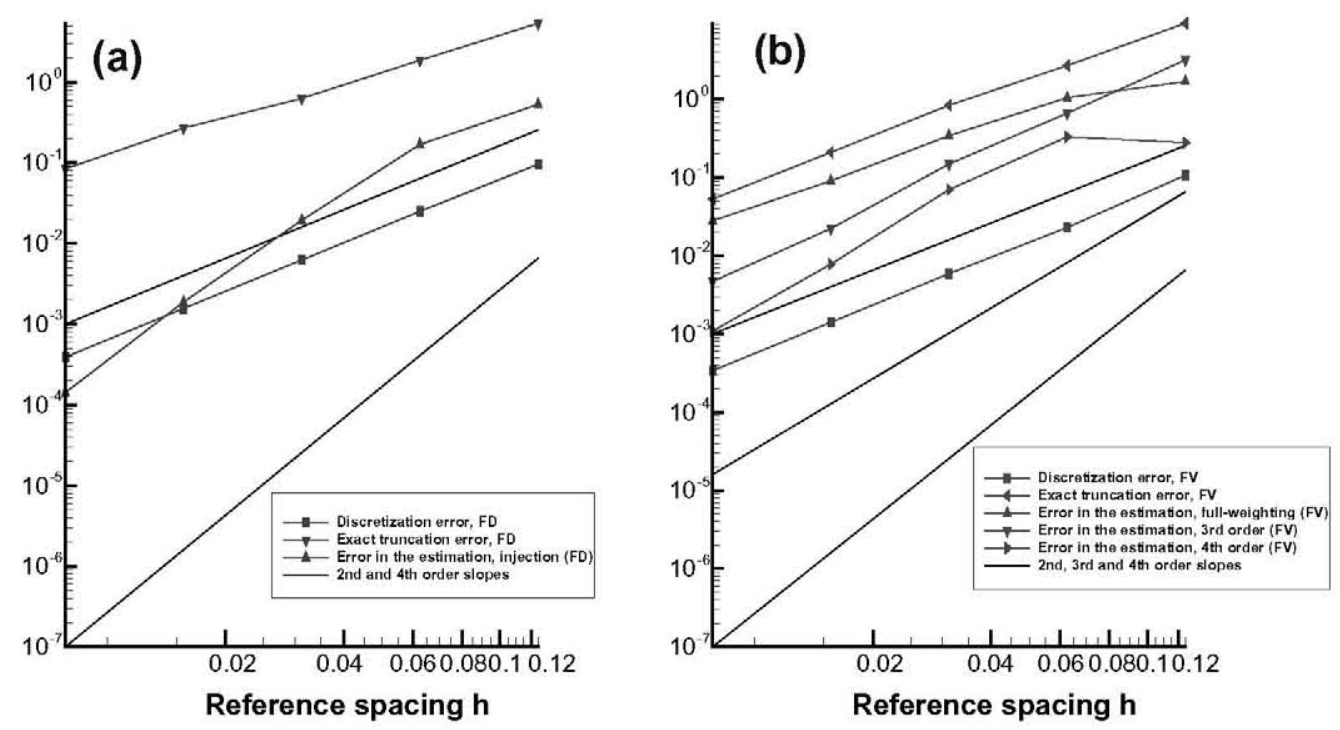

Fig. 8. $\|\cdot\|_{L_{\infty}}$ of the error in the truncation error estimate for the 1D linear problem on (a): smooth non-uniform grids using finite difference method, (b): smooth non-uniform grids using finite volume method.
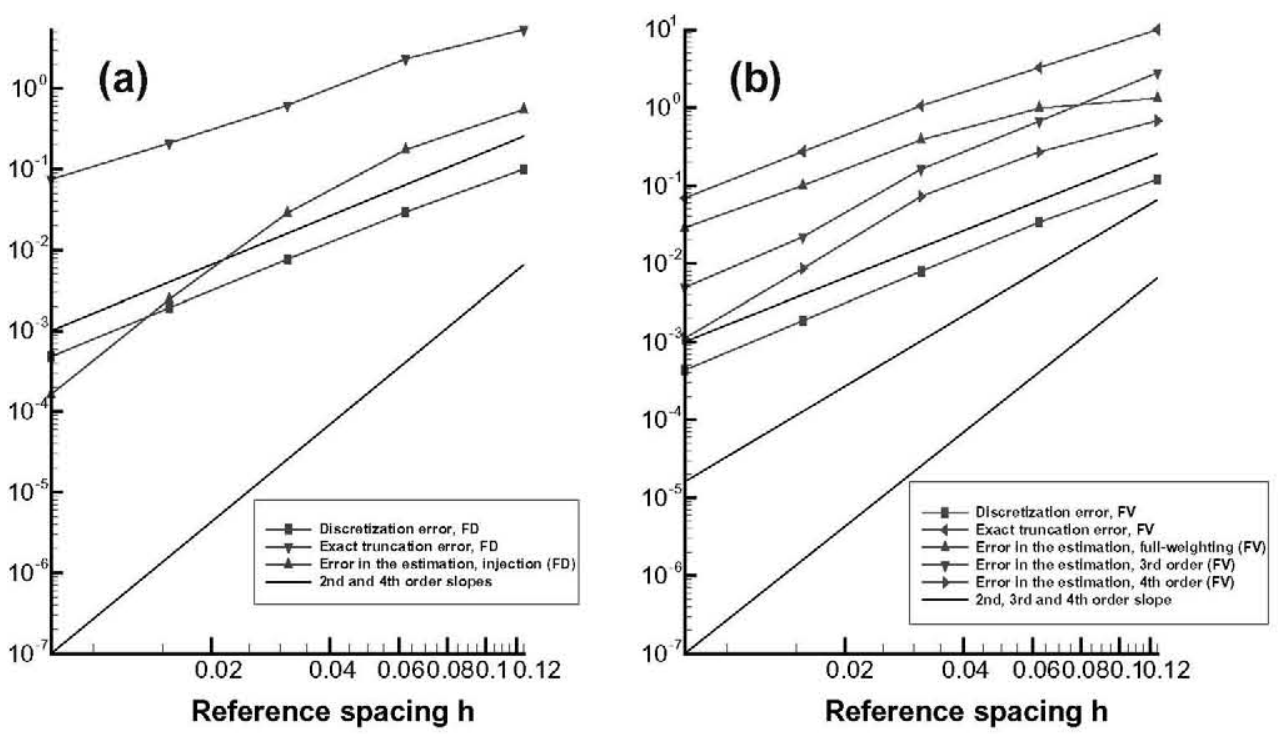

Fig. 9. $\|\cdot\|_{i_{\infty}}$ of the error in the truncation error estimate for the1D non-linear problem on (a): smooth non-uniform grids using finite difference method, (b): smooth non-uniform grids using finite volume method.

subgrids were extracted by removing one point every two points. Constructing the mesh levels in this way ensures that the quality of the grid improves from a coarse level to a fine level, which fulfils the requirements of Oberkampf [1] for a systematic grid refinement study. Conversely, as explained earlier, this strategy also allowed us to decrease the magnitude of the non-uniform/inconsistent terms of Eqs. (28) and (29) when the grid is refined.

The $L_{\infty}$ norm of the discretisation error, the exact truncation error and the error in the estimation $\left\|\tau^{H}-4 / 3 \tau_{h}^{H}\right\|_{\infty}$ have been computed, and they are reported in Figs. 8 and 9 for the linear and non-linear problems.

It is first remarkable that for this specific choice of grid distribution, as discussed before, both discretisation and truncation errors decrease at a second-order rate. Concerning the truncation error estimation, the conclusions are the same as for the uniform study in Section 3.1.1. A restriction of order $s>p$ is necessary to obtain an accurate estimation.

\section{Highly distorted grids}

Thus far, the estimation of the truncation error has been performed on uniform and smooth non-uniform meshes. It is interesting to observe what occurs when the grid becomes highly distorted, for example, if the truncation error estimation 


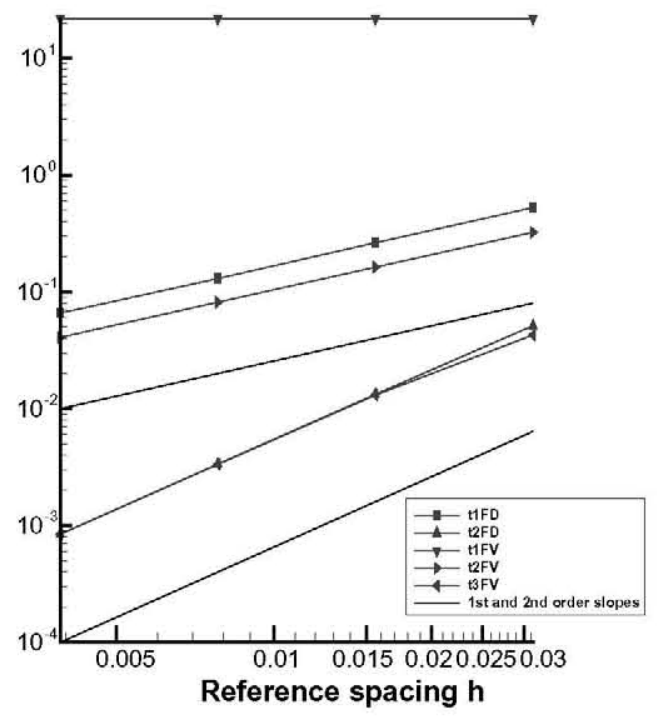

Fig. 10. $\|\cdot\|_{L_{\infty}}$ of the non-uniform terms in the truncation error expressions (Eqs. (28) and (29)) using grid distribution from Eq. (31).

must be performed on unstructured or hybrid grids when the quality of the original mesh is not optimal. For this purpose, we performed an analysis of the linear problem of Eq. (23) by using the following distribution of nodes:

$$
x_{i}=(i-1) h_{\text {unif }}+(\operatorname{rand}(i)-0.5) h_{\text {unif }}^{q}, \quad h_{\text {unif }}=\frac{1}{\operatorname{imax}-1}, \quad q \geqslant 1
$$

where the function rand returns a random value between 0 and 1 and $q$ controls the distortion of the mesh. Eq. (31) is composed of two parts; the first term on the right-hand side is the uniform distribution, whereas the second term represents a perturbation of order $\mathcal{O}\left(h^{q}\right)$ with a maximum amplitude $\pm 0.5 h_{\text {unij. }}$.

In this section, we studied the influence of the grid non-uniformities on the accuracy of the truncation error estimation. Thus, we did not consider a systematic mesh refinement that decreases the magnitude of the non-uniform terms in Eqs. (28) and (29). Instead, we built a sequence of successively refined meshes from an imax $=17$ base grid obtained from Eq. (31). The fine meshes were obtained by the bisection of coarse grid cells. In this manner, the characteristics of the base grid were maintained throughout the refinement process. Note that this approach is also valid in the sense of consistent mesh refinement (see Oberkampf [1]). As shown in Fig. 10 for $q=1$, the first term of the truncation error expression for the FV method (Eq. (29)) is of order unity. By contrast, the first term of the truncation error expression for the FD method (Eq. (28)) is of order one.

At each grid level, a coarse grid was extracted by removing one point every two points to compute the truncation error estimation of Eq. (6). Plots of the error committed in the estimation of the truncation error as a function of the parameter $q$ are reported in Fig. 11(a) for the FD method using injection to restrict the solution and in Fig. 11(b) for the FV method using fourth-order interpolation to restrict the solution.

Concerning the FD case, the truncation error magnitude decreases as $\mathcal{O}(h)$ for $q=1$ and $q=2$. Only when the distortion becomes very small ( $q=3$ ) does the truncation error decrease at a second-order rate. This naturally follows Eq. (28); for this sequence of grids, the truncation error is dominated by the first term of Eq. (29) (see Fig. 10), unless the distortion of the grid, governed by the parameter $q$, becomes negligible. The estimation of the truncation error, even for these low-quality grids, is highly accurate. In all cases, the magnitude of the error in the estimation $\left\|\tau^{H}-4 / 3 \tau_{h}^{H}\right\|_{\infty}$ decreases faster than the exact truncation error. Conversely, the discretisation error is not very dependent on the quality of the grid. The discretisation error decreases at a second-order rate for all $q$. This trend is due to the diffusion of the truncation error according to the error transport equation (Eq. (1)).

The case of FV is more subtle. As mentioned earlier, if no special care is taken in the initial grid node distribution and in the refinement process, the analytic expression of the truncation error (Eq. (29)) yields an inconsistent term that dominates (see Fig. 10). The inconsistent term decreases as $h^{q-1}$ on the base grid, and as shown in Fig. 11(b), is constant throughout mesh refinement; therefore, the assumption $p \geqslant 1$ of Section 2 is not valid, and the accuracy of the estimation cannot be guaranteed. This scenario does not invalidate the estimation of the truncation error in FV solvers for distorted grids. Instead, this result indicates the importance of a rigorous study of the consistency of the numerical scheme employed to compute the fluxes, as we demonstrated in this work that even a very simple FV discretisation might deteriorate in the presence of grid non-uniformities.

However, even for highly distorted grids, the estimator agrees with the FV method. Fig. 12(a)-(b) reports the exact truncation error and the estimation for $i \max =33$ and $i \max =257$ using $q=1$. Even for this pathological case, the main features are 


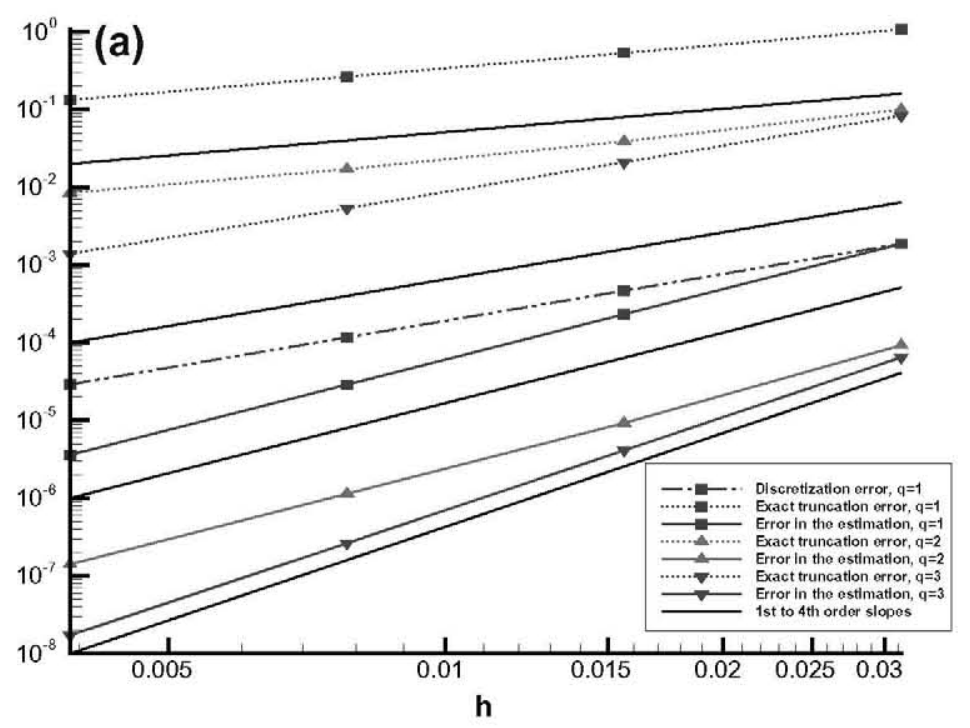

(b)

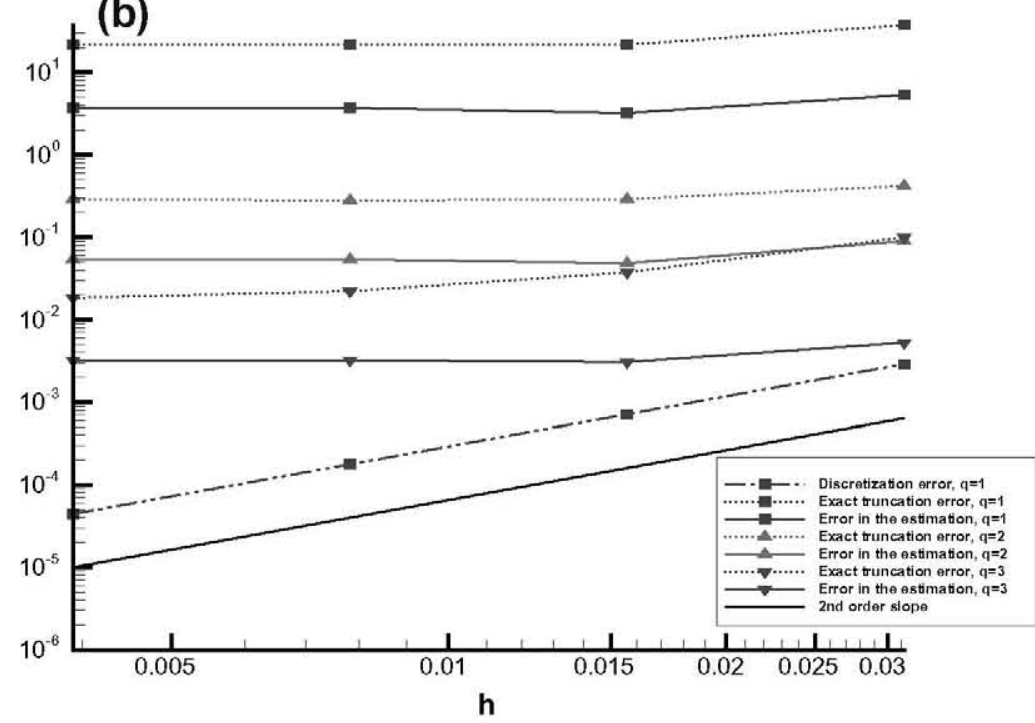

Fig. 11. Error in the truncation error estimate using distorted grids, (a): finite difference method, (b): finite volume method.

captured with reasonable accuracy. By contrast, this situation means that if the estimation is used as an indicator for mesh adaptation and if the initial grid is of very poor quality, the estimator will still predict the error with reasonable accuracy. Note that this is of importance when mesh adaptation in FV solvers, by local bisection of the edges, is performed. Also, in the regions where the magnitude of the truncation error is of order unity (for example, near discontinuities), the estimator is expected to maintain a reasonable behaviour. We experimented with the latter on a shocked flow in Section 4 .

\subsubsection{Effect of iteration error}

The previous analysis has considered the case of a converged solution. In fact, most papers in the literature (Berger [18], Bernert [19] or Fulton [20]) were devoted to the estimation of the truncation error on converged solutions. However, in Section 2, we derived the full truncation error estimate expression (Eqs. (7) and (8)) where the iteration error $\epsilon_{i t}^{h}$ is introduced. For the linear case, this derivation yielded a condition to be fulfilled for an accurate estimation of the truncation error when the solution has not converged (Eq. (17)). We propose in this section to verify this equation for the one-dimensional linear problem on uniform grids, and we discuss its extension to general problems. The present study was performed using the FD method on a uniform one-dimensional grid.

For convenience, we rewrote Eq. (17) as follows:

$$
\mathcal{I}_{h}^{H}=\mathcal{L}_{\text {dca }}^{H} \widehat{\mathcal{I}}_{h}^{H}\left(\mathcal{L}^{h}\right)^{-1}
$$



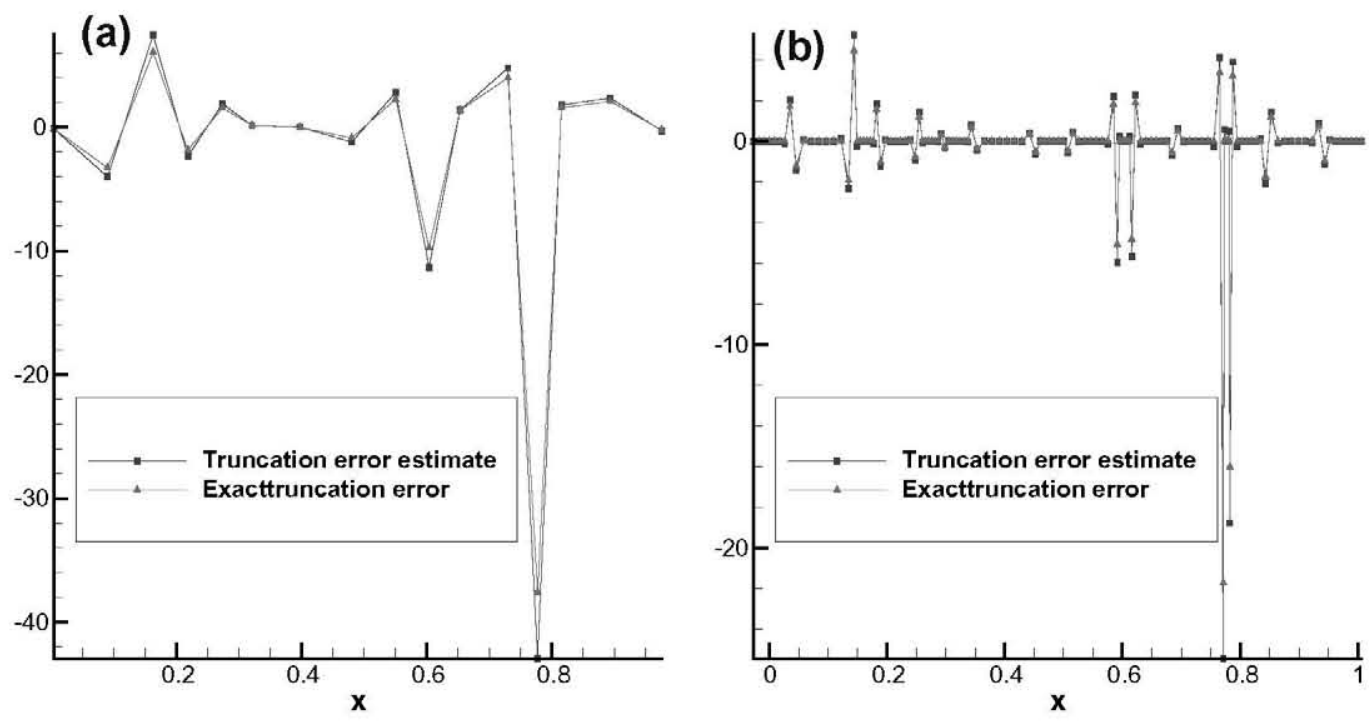

Fig. 12. Estimation of the truncation error for highly distorted mesh, $q=1$ for finite volume method, (a): imax =33, (b): imax $=257$.

Table 2

Linear restriction operators satisfying Eq. (17).

\begin{tabular}{|c|c|c|c|c|c|c|c|}
\hline Index fine grid & $i-3$ & $i-2$ & $i-1$ & $i$ & $i+1$ & $i+2$ & $i+3$ \\
\hline$h^{2} \mathcal{L}^{h}$ & 0 & 0 & -1 & 2 & -1 & 0 & 0 \\
\hline$H^{2} \mathcal{L}_{d c a}^{H}$ & 0 & -1 & 0 & 2 & 0 & -1 & 0 \\
\hline$\hat{\mathcal{I}}_{h}^{H}=$ injection & 0 & 0 & 0 & 1 & 0 & 0 & 0 \\
\hline Corresponding $\mathcal{I}_{h}^{H}$ & 0 & 0 & 0.25 & 0.5 & 0.25 & 0 & 0 \\
\hline$\hat{\mathcal{I}}_{h}^{H}=3 \mathrm{rd}$ order & 0 & 1 & -2 & 1 & 2 & -1 & 0 \\
\hline Corresponding $\mathcal{I}_{h}^{H}$ & 0.25 & 0 & -0.5 & 0.5 & 1 & 0 & -0.25 \\
\hline$\hat{\mathcal{I}}_{h}^{H}=4$ th order & 0 & 1 & -4 & 7 & -4 & 1 & 0 \\
\hline Corresponding $\mathcal{I}_{h}^{H}$ & 0.25 & -0.5 & 0 & 1.5 & 0 & -0.5 & 0.25 \\
\hline
\end{tabular}

This equation states that, for a given linear discrete operator $\mathcal{L}^{h}$ (we remind the reader that $\mathcal{L}_{\text {dca }}^{H}$ is the coarse grid representation of $\mathcal{L}^{h}$ ) and a given linear restriction operator $\widehat{\mathcal{I}}_{h}^{H}$, a linear residual transfer operator $\mathcal{I}_{h}^{H}$ permits eliminating the remaining terms of Eq. (7). Eq. (32) can be inverted to derive a restriction operator for the solution $\widehat{\mathcal{I}}_{h}^{H}$ from a given transfer operator for the residual $\mathcal{I}_{h}^{H}$ and the discrete PDE operator $\mathcal{L}_{d c a}^{H}$. However, for our purposes, we prefer the formulation of Eq. (32), as we have constraints on the order of accuracy $s$ (see Section 2) for the restriction acting on the solution, whereas no other constraints apply to the restriction of the residual $\mathcal{I}_{h}^{H}$.

By applying Eq. (32) to three solution restriction operators (injection, third and fourth orders), we obtained the following residual transfer operators, written in Table 2 in stencil notation. The symmetry of the problem allowed us to consider only $7 \times 7$ matrices in Eq. (32), thus making the resolution independent of the overall number of nodes.

From Table 2, the injection/full-weighting for the solution and the residual, which is a common combination within the multigrid strategy, in theory provides an accurate estimation of the truncation error at the first iteration. When the thirdand fourth-order operators are used to transfer the solution, residual operators also exist that, in theory, allow us to eliminate the remaining terms of Eq. (7).

Next, we propose to verify that the restriction operators from Table 2 truly cancel out the terms containing the iteration error in Eq. (7). For this purpose, we computed the truncation error estimate on the linear one-dimensional problem (Eqs. (23) and (24)) using the FD method and a uniform grid composed of 129 nodes. Each pair of restriction operators from Table 2 has been tested, and the results are reported in Fig. 13(a), which reports the exact truncation error $\left\|\tau^{H}\right\|_{\infty}$ and the error in the estimation $\left\|\tau^{H}-4 / 3 \tau_{h}^{H}\right\|_{\infty}$. The results are compared with results obtained with the same restriction operators for the solution, but using injection for the residual instead of the operators computed with Eq. (32). These results are in perfect agreement with our analysis. When injection is used to restrict the residual, it does not fulfil the requirement Eq. (32) to obtain an accurate estimation in the first iteration. This relationship can be observed by deriving the remaining terms of Eq. (7), as follows. 
The first term (where the superscripts denote grid indices, for convenience), using injection for the restriction $\mathcal{I}_{h}^{H}$, reads as follows:

$$
\mathcal{I}_{h}^{H} \mathcal{L}^{h} \epsilon_{i t}^{h}=\frac{-\epsilon_{i t}^{i+1}+2 \epsilon_{i t}^{i}-\epsilon_{i t}^{i-1}}{h^{2}}
$$

The second term, which also uses injection to restrict the solution, becomes

$$
\mathcal{L}^{H} \hat{\mathcal{I}}_{h}^{H} \epsilon_{i t}^{h}=\frac{-\epsilon_{i t}^{i+2}+2 \epsilon_{i t}^{i}-\epsilon_{i t}^{i-2}}{4 h^{2}}
$$

Then, combining Eqs. (33) and (34) yields

$$
\mathcal{I}_{h}^{H} \mathcal{L}^{h} \epsilon_{i t}^{h}=\mathcal{L}^{H} \hat{\mathcal{I}}_{h}^{H} \epsilon_{i t}^{h}+\frac{h^{2}}{4} \frac{\partial^{4} \epsilon_{i t}}{\partial x^{4}}+\mathcal{O}\left(h^{4}\right)
$$

Then, Eq. (18) holds with $c=1 / 4, l=2$ and $m=4$.

In this case, even if the estimation is not accurate in the first relaxation steps, the estimation converges to the case where the restriction operator is computed with Eq. (32). This result is clear because, as long as the solution is advanced in pseudotime, the residual tends to equal zero, and thus the second term of Eq. (6) vanishes.

Another remark concerns the curve corresponding to the third-order restriction for the solution in combination with the restriction operator for the residual given in Table 2 . It can be noted that its magnitude does not appear to be much lower than the actual exact truncation error. However, we remind the reader that this result is a single grid result, and it can be verified that this third-order restriction operator on a sequence of grids of different spacing will exhibit third-order behaviour of the error in the estimation $\left\|\tau^{H}-4 / 3 \tau_{h}^{H}\right\|_{\infty}$, as predicted in the analysis of Section 2 .

We have discussed the simplest case using FD, linear problems and uniform grids. Although the results are very encouraging, we shall discuss the extension to other discretisations.

- The extension to a linear FV problem is straightforward when a uniform grid is concerned because both methods yield the same discretisation.

- The extension to non-uniform grids does not pose theoretical issues. We expected to obtain an a priori error estimation. However, in practice, the symmetry of the discrete PDE operators is lost, thus solving Eq. (32) cannot be accomplished by considering a single $7 \times 7$ matrix, independent of the overall number of nodes. In this case, local inversions must be performed.

- Finally, the extension to non-linear problems is clearly more subtle. Some precise analyses should be performed on the structure of the Jacobian. It is also important to note that in the analysis of Section 2, the assumption $\epsilon_{i t}^{h} \ll \mathcal{I}^{h} u$ has been made to derive Eq. (8). Thus, it is a difficult task to conclude the existence of restriction operators or coarse grid discrete PDE operators, which allow us to completely eliminate the remaining terms of Eq. (8) before any relaxation is applied. We left this exploration for future work. We proposed instead to test the restriction operators computed for the linear case (from Table 2) on the non-linear problem. Although a finer analysis would be required to ensure that they are optimum for the minimisation of the remaining terms of Eq. (8), we can see in Fig. 13(b) that they allow for an accurate truncation error estimation far before the solution has converged. For example, using injection/full-weighting (which satisfies Eq. (32) for the linear problem) to restrict the solution and the residual, the error in the estimation $\left\|\tau^{H}-4 / 3 \tau_{h}^{H}\right\|_{\infty}$ is two orders of magnitude lower than the exact truncation error for a relatively high residual norm of $2.10^{-1}$. Although a finer analysis would be necessary, this preliminary result for the non-linear equation demonstrates that the solution does not need to be fully converged to obtain a reasonable accuracy in the truncation error estimate.

These results are encouraging and of importance for many applications; for example, in dynamic mesh adaptation, CPU time might be saved if the estimation of the truncation error is used to flag regions for refinement. This strategy might be particularly useful with meshes of bad quality because an estimation of the truncation error would be available in only a few iterations.

\subsection{Two-dimensional test cases}

The analysis performed for one-dimensional equations was extended in this section on two-dimensional problems. The analysis of Section 2 does not make any assumptions on the dimensionality of the problem. However, it states that the discretisation and truncation error have the same formulation both on coarse and fine mesh, requiring, as we demonstrate later in this section, some consistency in the topology of both grids. Therefore, under the assumption of topologically similar grids, we indicate in this section that the extension to two-dimensional partial differential equations is straightforward.

As in the previous case, the 2D linear diffusion (Poisson equation) and the nonlinear convection-diffusion (Burger's equations) are studied. The $2 \mathrm{D}$ equations considered here are as follows:

$$
-\Delta u=f_{1}+\text { b.c. }, \quad-\Delta u+u u_{x}=f_{2}+b . c
$$



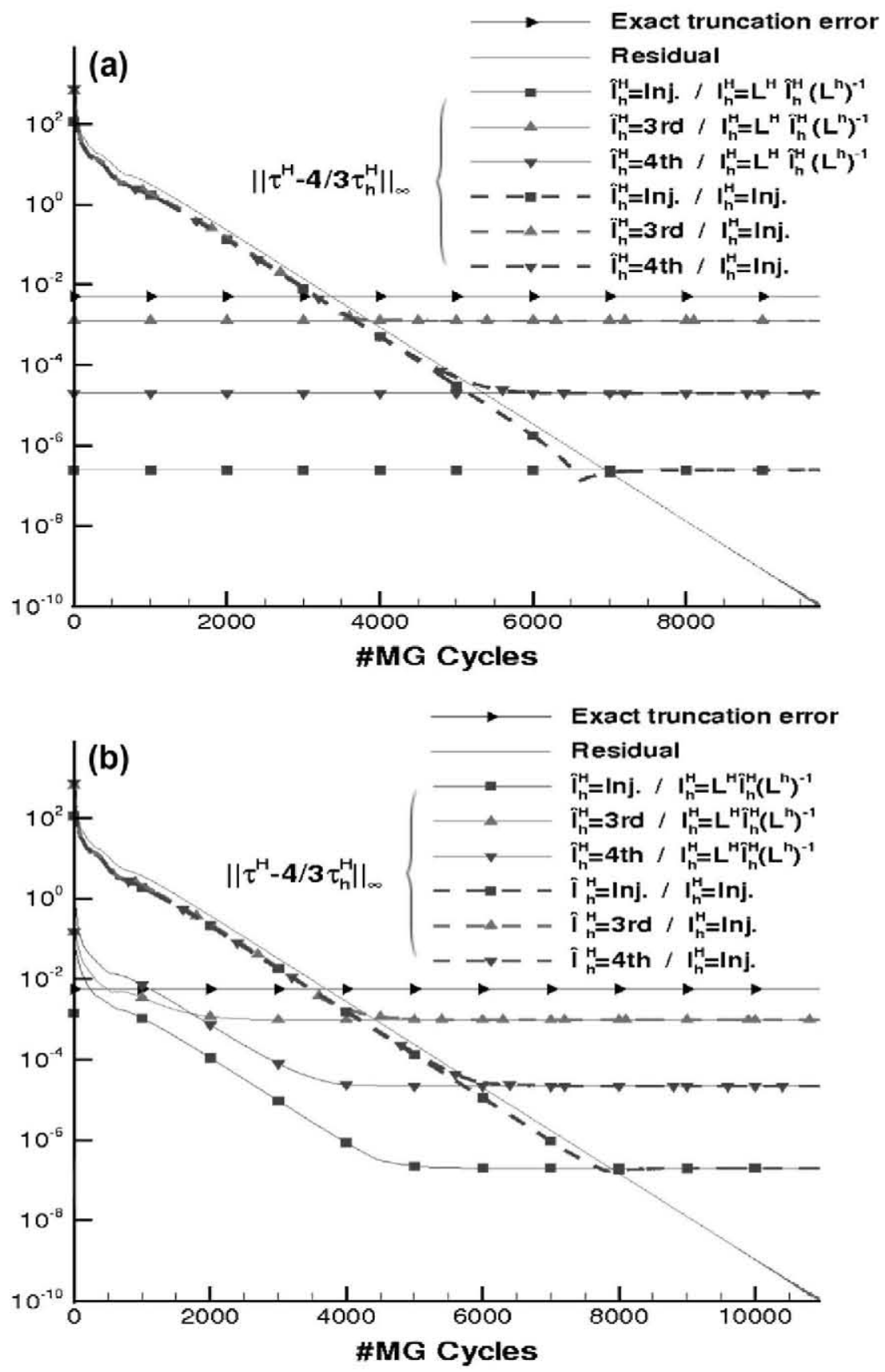

Fig. 13. Evolution of the accuracy of the estimator, for $1 \mathrm{D}$ finite difference problem on imax $=129$ uniform grid, with respect to the convergence process. (a) Linear problem and (b) non-linear problem.

where b.c represents boundary conditions. We considered the following test functions:

$$
\left\{\begin{array} { l } 
{ f _ { 1 } ( x , y ) = 5 2 \operatorname { c o s } ( 4 x + 6 y ) } \\
{ u ( x , y ) = u _ { e x } ( x , y ) , \forall ( x , y ) \in \Omega = [ 0 , 1 ] ^ { 2 } }
\end{array} \quad \left\{\begin{array}{l}
f_{2}(x, y)=52 \cos (4 x+6 y)-4 \sin (4 x+6 y) \cos (4 x+6 y) \\
u(x, y)=u_{e x}(x, y), \forall(x, y) \in \Omega=[0,1]^{2}
\end{array}\right.\right.
$$

These problems have the following exact solution:

$$
u_{e x}(x, y)=\cos (4 x+6 y) \text {. }
$$

Eqs. (36) and (37) were solved using standard second-order spatial schemes. An FV method with a second-order central scheme for the computation of the fluxes was used. This spatial discretisation then yielded a second-order discretisation 


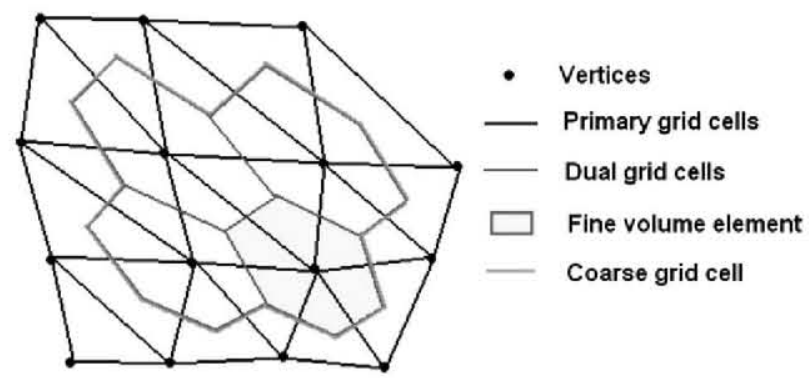

Fig. 14. Example of two-dimensional triangulated region. The coarse cell is obtained by fusion of the four fine elements.

error as well as a second-order truncation error on smooth meshes. The steady-state solution was reached using a RungeKutta relaxation scheme and a multigrid strategy.

Similar to the one-dimensional case, we studied the effect of the restriction operator, acting on the converged solution, on the accuracy of the estimator. No further investigations are presented on the boundary conditions, the non-uniform grids and the iteration errors, as the conclusions were similar. Instead, we developed the case of fine and coarse grids possessing distinct topologies.

\subsubsection{Role of the restriction operator}

Fig. 15 reports the error in the estimation of the truncation error for linear, non-linear, and for both uniform and smooth non-uniform quadrilateral meshes (using the distribution of nodes of Eq. (30)). The conclusions are similar to the one-dimensional case; when second-order full weighting is used to restrict the solution, the estimator is not accurate. When third-and fourth-order interpolation are used, the error in the estimation $\left\|\tau^{H}-4 / 3 \tau_{h}^{H}\right\|_{\infty}$ is of the order of three and four, respectively, which ensure the estimation to be accurate, as the exact truncation error is of the order of two.

\subsubsection{Topology concerns}

Thus far, estimations of the local truncation error have been obtained using a set of structured grids, coarsened equally in each direction. However, in the case of Cartesian grids and if we discretise the linear Eq. (36) using a central scheme, the expression of the truncation error has two components decoupled in the $x$ and $y$ directions. In this case, it is possible to coarsen the fine grid in only one direction and then obtain an estimate of the truncation error along one direction only. Indeed, let us consider $\Omega^{h}$ to be a uniform grid of spacing $\Delta x, \Delta y$ and consider $\Omega^{H}$ to be the associated coarse grid of spacing $2 \Delta x, \Delta y$. We then have the following equation:

$$
\begin{aligned}
\tau^{h} & =-\frac{\Delta x^{2}}{12} \mathcal{I}^{h} \frac{\partial^{4} u}{\partial x^{4}}-\frac{\Delta y^{2}}{12} \mathcal{I}^{h} \frac{\partial^{4} u}{\partial y^{4}} \\
\tau^{H} & =-\frac{4 \Delta x^{2}}{12} \mathcal{I}^{H} \frac{\partial^{4} u}{\partial x^{4}}-\frac{\Delta y^{2}}{12} \mathcal{I}^{H} \frac{\partial^{4} u}{\partial y^{4}}
\end{aligned}
$$

Then, restricting $\tau^{h}$ using full weighting yields

$$
\tau^{H}-\mathcal{I}_{h}^{H} \tau^{h}=-\frac{3}{4} \frac{(2 \Delta x)^{2}}{12} \mathcal{I}^{H} \frac{\partial^{4} u}{\partial x^{4}}+\mathcal{O}\left(h^{4}\right)
$$

which is the component of the truncation error in the $x$ direction. Therefore, the analysis of Section 2 holds, and an accurate estimation of this contribution can be performed by the use of Eq. (7).

However, the latter is a specific case. An important issue to tackle with 2D/3D geometries is that the estimator presented in this work makes the assumption that the formulation of the truncation error for a given operator $\mathcal{L}$ only varies with the mesh spacing (assumption (A1)). This assumption is true for an FV formulation if the fine and coarse mesh share the same topology and the same type of elements. Indeed, if we develop Eq. (3) for the linear Poisson equation Eq. (36) on arbitrary elements, we obtain

$$
\tau^{h}=\mathcal{L}^{h} \mathcal{I}^{h} u-f^{h}=-\frac{1}{V^{h}} \sum_{i \in \partial \Omega^{h}}\left(\left(\vec{\nabla}^{h} \mathcal{I}^{h} u\right)_{\partial S_{i}^{h}}+\vec{\tau}_{\vec{\nabla}_{i}^{h}}^{h}\right) \cdot \vec{n}_{\partial \Omega_{i}^{h}}^{h} S_{\partial \Omega_{i}^{h}}^{h}-f^{h}=-\frac{1}{V^{h}} \sum_{i \in \partial \Omega^{h}} \vec{\tau}_{\nabla_{i}}^{h} \cdot \vec{n}_{\partial \Omega_{i}^{h}}^{h} S_{\partial \Omega_{i}^{h}}^{h}+\mathcal{O}\left(\left(S_{\partial \Omega_{i}^{h}}^{h}\right)^{3}\right)
$$

where $V_{h}$ is the volume of the cell with discrete boundaries $\partial \Omega_{j}^{h}, \vec{\nabla}^{h}$ is the discretised gradient operator with the associated truncation error $\vec{\tau}_{\vec{b}^{h}}^{h}, \vec{n}_{\partial \Omega_{i}^{h}}^{h}$ is the unit normal to the face and $S_{\partial \Omega_{i}^{h}}^{h}$ is the surface area.

Overlined symbols represent averaging (typically at the centre of the faces), and then, the third-order term arises from this averaging.

The presence of the normals to the faces is illustrated in Eq. (38). When the same type of elements are used for the fine and coarse grids, these normals cancel each other when taking the difference of the fine and coarse truncation errors, and the 

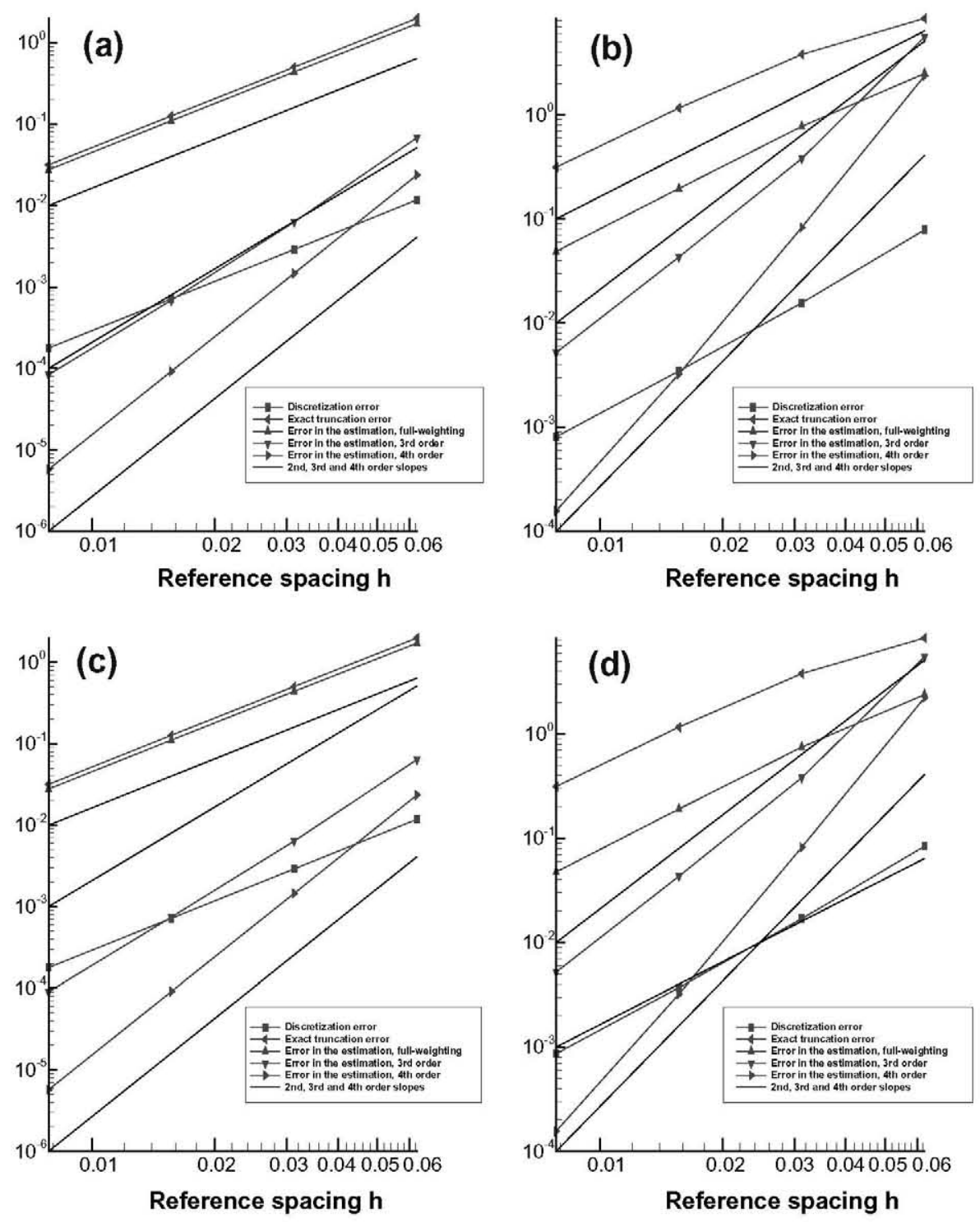

Fig. 15. $\|\cdot\|_{i}$ of the error in the truncation error estimate for the 2D problems. (a) Linear problem on uniform grids, (b) linear problem on smooth nonuniform grids, (c) non-linear problem on uniform grids and (d) non-linear problem on smooth non-uniform grids.

assumption (A1) holds. However, when different elements are used (for example, if coarse grids are generated by the agglomeration of fine grid cells, for example, the agglomeration of the four dual cells of Fig. 14) on the fine and the coarse grids, the truncation error differs not only by the grid spacing but also by the orientation of the elements; then, the analysis of Section 2 does not hold. This fact might be of importance if the method is to be applied on agglomeration-based multigrid solvers. Therefore, maintaining consistency in the topology from the fine grid to the coarse grids is mandatory as far as the $\tau$ estimation is concerned. We discuss this issue using an example in Section 4.

\section{Numerical experiments on two-dimensional Euler equations}

We finished our study by performing an analysis of the estimation of the truncation error on the two-dimensional Euler equations, as follows:

$$
\frac{\partial \mathbf{U}}{\partial t}+\frac{\partial \mathbf{F}}{\partial x}+\frac{\partial \mathbf{G}}{\partial y}=\mathbf{S}
$$


with

$$
\mathbf{U}=\left(\begin{array}{c}
\rho \\
\rho u \\
\rho v \\
\rho E
\end{array}\right), \quad \mathbf{F}=\left(\begin{array}{c}
\rho u \\
\rho u^{2}+p \\
\rho u v \\
\rho u H
\end{array}\right), \quad \mathbf{G}=\left(\begin{array}{c}
\rho v \\
\rho u v \\
\rho v^{2}+p \\
\rho v H
\end{array}\right)
$$

and

$$
p=(\gamma-1)\left(\rho E-\frac{\rho\left(u^{2}+v^{2}\right)}{2}\right)
$$

Here, $\mathbf{S}$ is a source term introduced in the context of the method of manufactured solutions, and it is discussed in Section 4.1.

We solved Eqs. (39) and (40) on quad- and triangle-based geometries using the vertex-based FV DLR TAU-Code [22]. DLR TAU-Code solves the Reynolds Averaged Navier-Stokes equations on unstructured hybrid grids by employing a second-order FV discretisation. The multigrid strategy implemented in TAU uses the full approximation scheme algorithm to compute the correction term on the coarse grids. The coarse grids are obtained by agglomeration of the fine grid dual cells. When the primary grid is composed of quadrilaterals (or hexahedrals for 3D computations), then the advanced front method is capable of agglomerating 4 quadrilaterals ( 8 hexahedrals) to create a coarse quadrilateral (hexahedral), as in a structured solver. However, when the primary grid is unstructured, the agglomeration algorithm creates coarse grid elements that do not necessarily maintain the fine grid characteristics. In the context of multigrids, this situation is not a strong limitation, particularly in TAU, where the coarse grid fluxes are computed with a first-order accuracy. However, in the context of truncation error estimation, as we discussed earlier, it is of importance that the truncation error is identical between fine and coarse grids. This goal clearly cannot be accomplished if the elements differ from fine to coarse mesh. To circumvent this issue and to obtain estimations of the truncation error for unstructured grids, in the following analysis, the fine mesh (where the flow solution is actually computed) was obtained from the coarse mesh by bisecting all of the edges. This method also allows the use of injection to restrict the solution from fine to coarse grids.

(a)

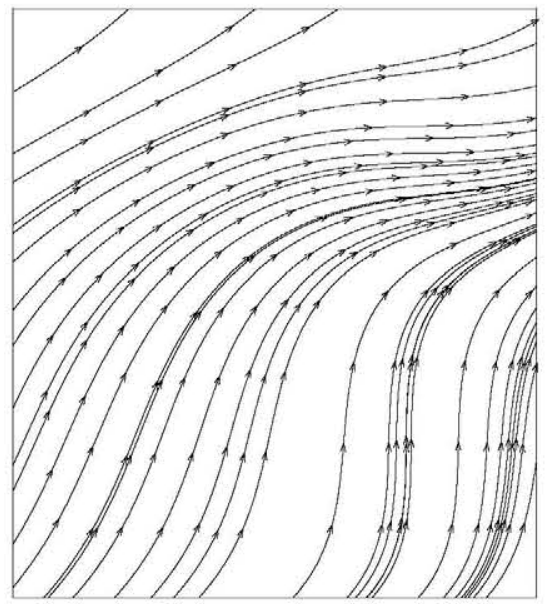

(c)

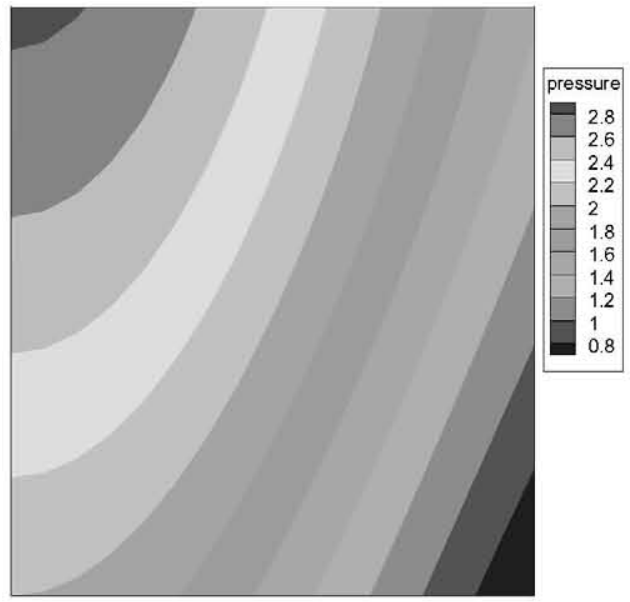

(b)

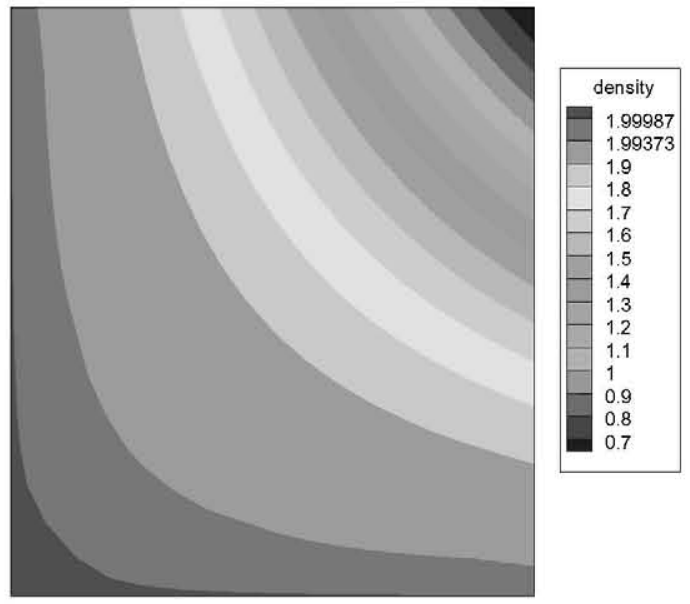

(d)

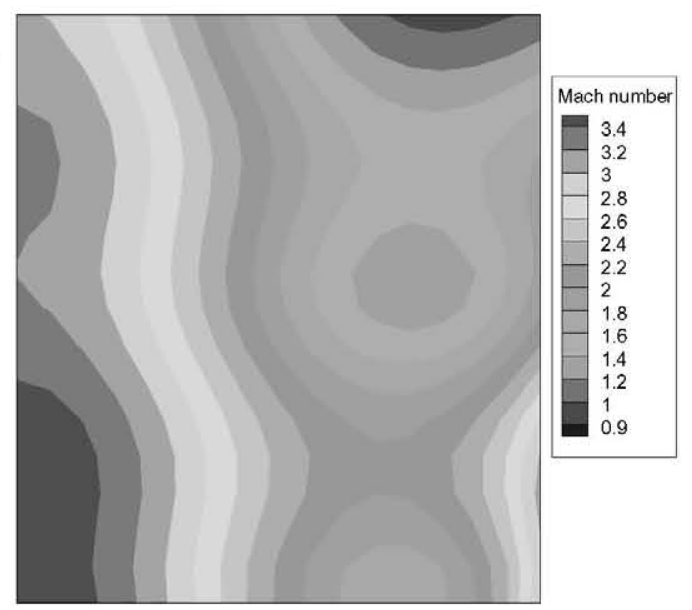

Fig. 16. Manufactured solution. (a) Velocity streamlines, (b) density, (c) pressure and (d) Mach number. 
Two different test cases are considered next, with and without discontinuities. The first test case was obtained by the method of manufactured solutions [2,24]. The second problem treated here is the supersonic confluent flow [7], which exhibits strong discontinuities. In both cases, the AUSMDV second-order upwind scheme [25] was employed using a least-squares approach to reconstruct the gradients at the edges.

\subsection{Method of manufactured solutions}

In the code verification step, when systematic mesh refinement studies are performed to examine the formal order of accuracy, it is important that the analytic solution employed fulfils some requirements. Although the form of the manufactured solution is somewhat arbitrary, it should be chosen to be smooth, infinitely differentiable (to avoid the cancellation of higher order derivatives) and realisable (i.e., solutions should be avoided that have negative densities, pressures, and temperatures). The solution chosen in the scope of this work is as follows:

$$
\begin{aligned}
& \rho(x, y)=1+\cos (2 x y) \\
& u(x, y)=2+\cos (4 x)-\sin (6 y) \\
& v(x, y)=2+\cos (4 x)+\sin (6 y) \\
& p(x, y)=1+\cos (2 x)+\sin (y)
\end{aligned}
$$
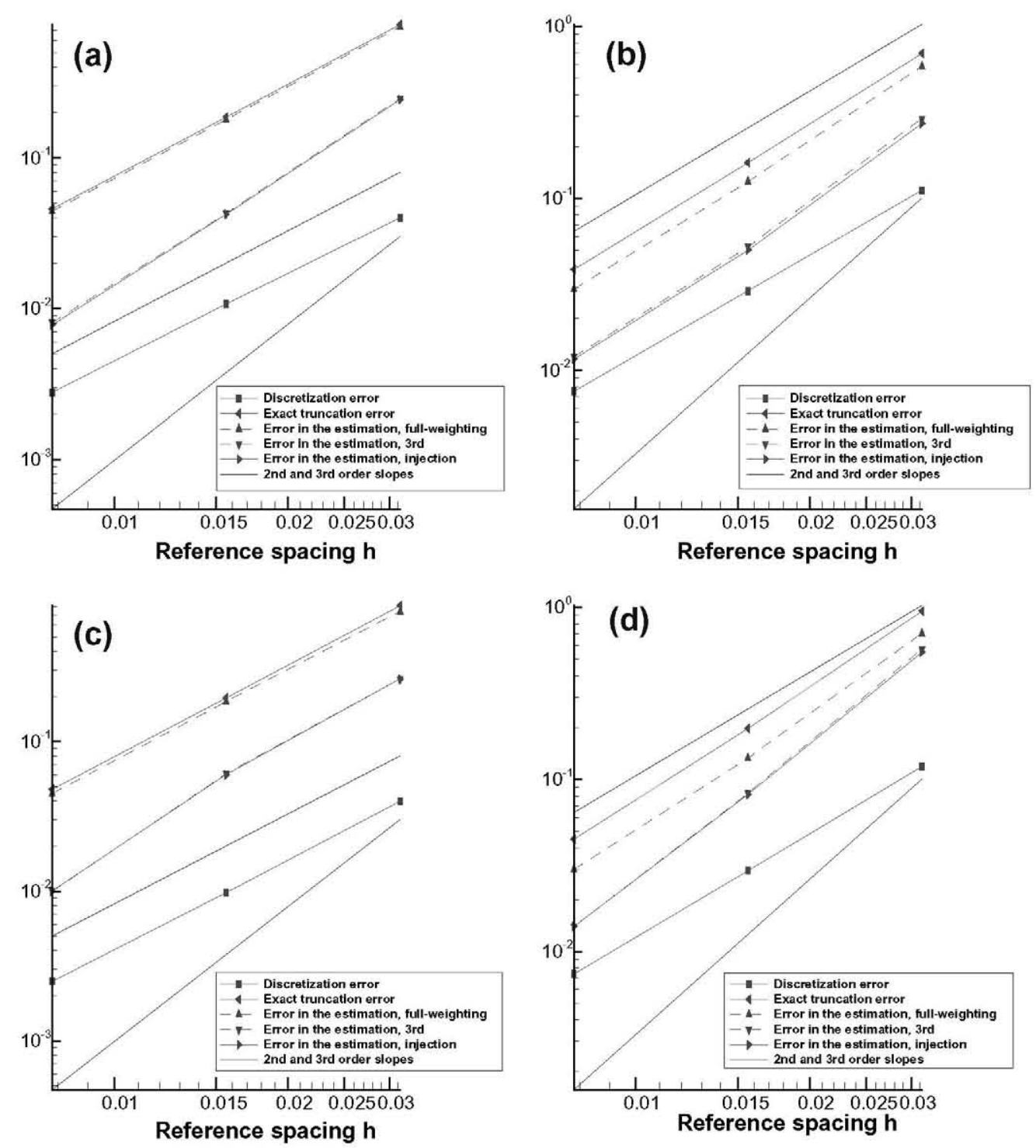

Fig. 17. $\|\cdot\|_{L_{2}}$ of the error in the truncation error estimate for the Euler MMS problem. (a) Quads-based uniform grids, (b) quads-based smooth non-uniform grids, (c) triangules-based uniform grids and (d) triangules-based smooth non-uniform grids. 
This set of manufactured solutions is infinitely differentiable; thus, no derivatives vanish. The solutions are sufficiently smooth to guarantee a rapid convergence towards the asymptotic rate but also exhibit locally higher gradients, as can be observed in Fig. 16. Obviously, this set of solutions does not satisfy the original Euler equations. To accomplish this, source terms must be added according to the manufactured solutions. This task can be performed by the use of symbolic differentiation and automatic code generation, as suggested by Roache [2]. For example, by inserting Eq. (41) into the Euler equations, the following source terms for the continuity and $x$-momentum equations arise:

$$
\begin{aligned}
S_{\rho}=- & 2 y \sin (2 x y)(2+\cos (4 x)-\sin (6 y))-4 \sin (4 x)(1+\cos (2 x y)) \\
& -2 x \sin (2 x y)(2+\cos (4 x)+\sin (6 y))+6 \cos (6 y)(1+\cos (2 x y)) \\
S_{\rho u}= & -2 y \sin (2 x y)(2+\cos (4 x)-\sin (6 y))^{2}-8 \sin (4 x)(2+\cos (4 x)-\sin (6 y))(1+\cos (2 x y)) \\
& -2 \sin (2 x)-2 x \sin (2 x y)(2+\cos (4 x)-\sin (6 y))(2+\cos (4 x)+\sin (6 y)) \\
& -6 \cos (6 y)(1+\cos (2 x y))(2+\cos (4 x)+\sin (6 y))+6 \cos (6 y)(1+\cos (2 x y))(2+\cos (4 x)-\sin (6 y))
\end{aligned}
$$

The estimation of the truncation error has been performed using second-and third-order restriction operators to transfer the solution from fine to coarse meshes. Only steady solutions are considered in the following, and thus, the influence of the iteration error is not studied. The third-order restriction operator needs the computation of second derivatives. This computation is performed by applying the least-squares method (already used for the computation of the first derivatives in the reconstruction of the fluxes for the upwind schemes) again on the first derivatives. Eq. ( 8 ) holds with $p=2, q=1$ and $s=2,3$ for second-and third-order restriction operators, respectively, and $\epsilon_{i t}^{h}=0$. The use of a sequence of embedded meshes (by edge bisection) allowed us to compare the results obtained using the second- or third-order restriction operators with an

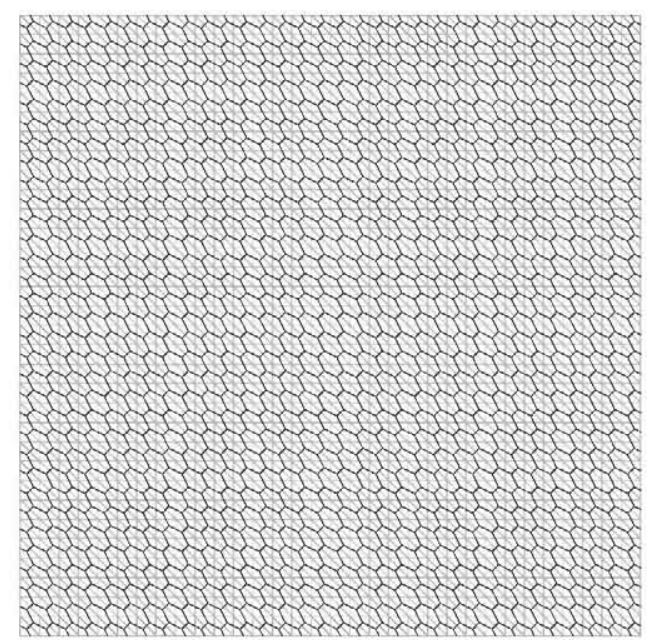

(a)

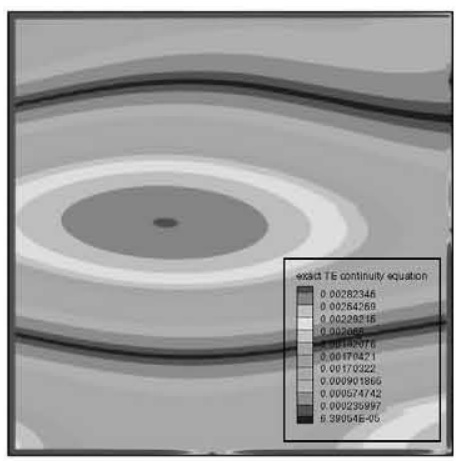

(c)

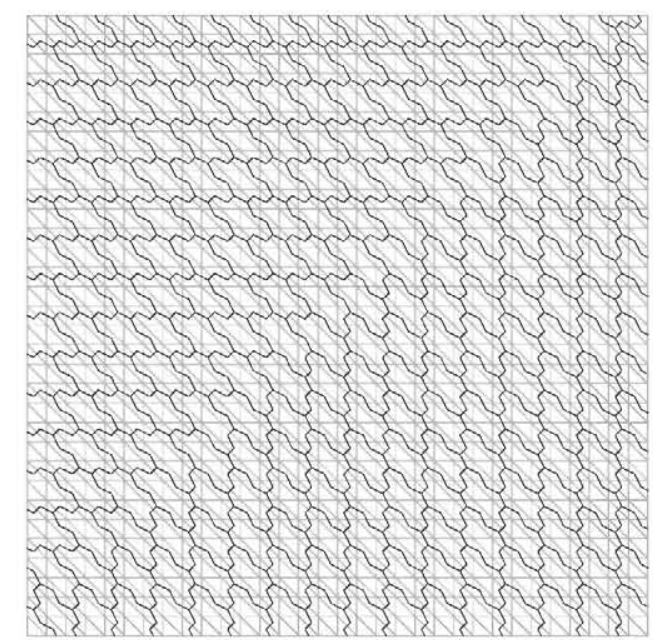

(b)

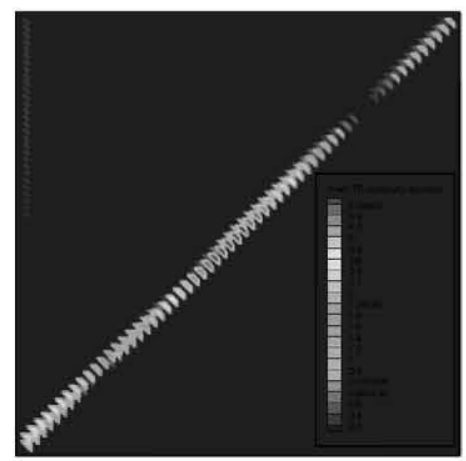

(d)

Fig. 18. Absolute value of the exact truncation error, continuity equation, on agglomerated coarse grid. (a) Close view of fine grid, (b) Close view of coarse agglomerated grid, (c) TE contours on the fine grid and (d) TE contours on the agglomerated grid. 

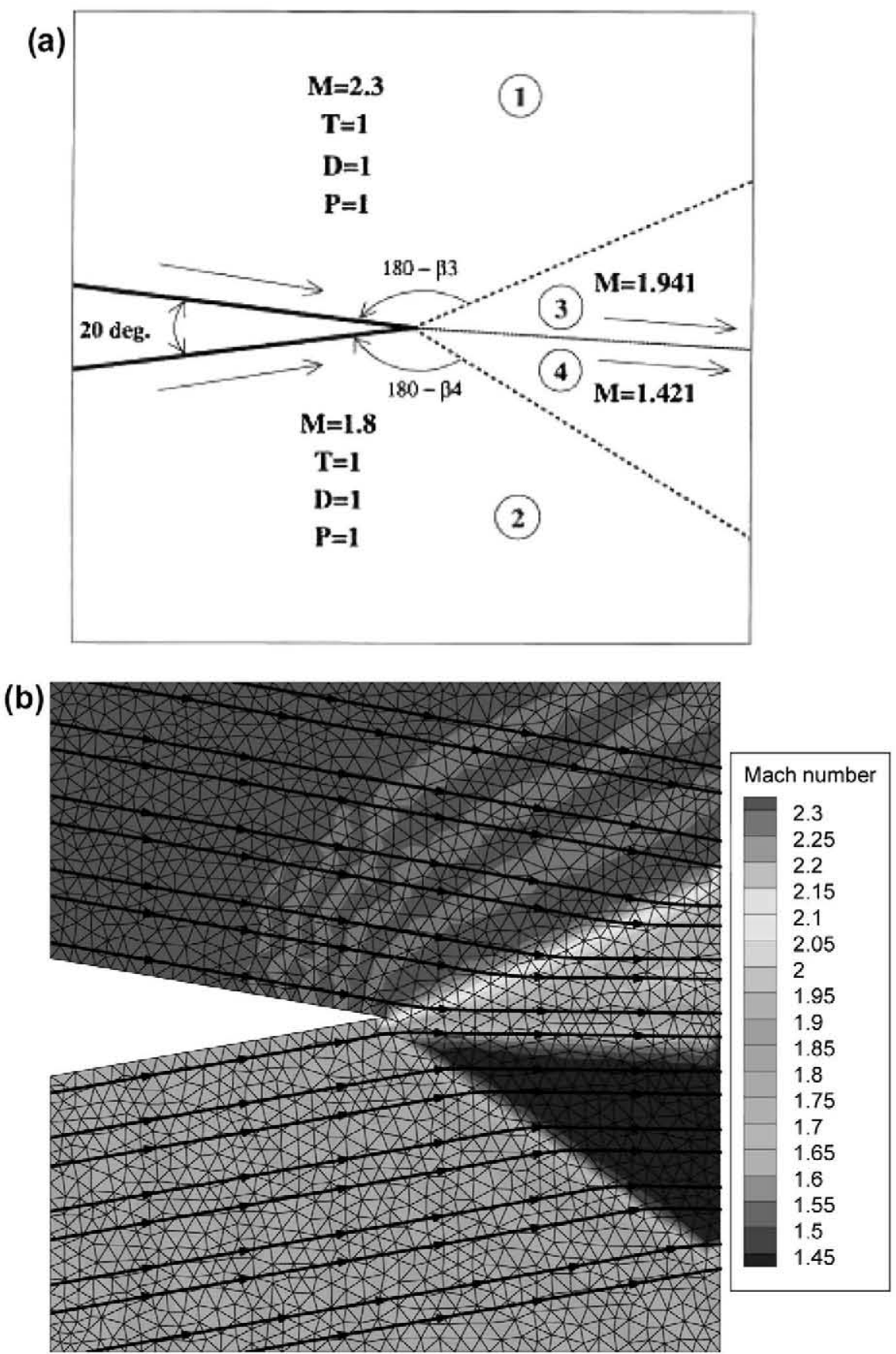

Fig. 19. Supersonic confluence flow. (a) Analytic solution and geometry and (b) numerical solution, Mach number contours and streamlines.

injection operator. Two primary grid elements are tested in the following, using quadrilaterals and triangulars, with the latter by the use of uni-directional diagonal meshes (destructured meshes).

The $L_{2}$ norm of the error in the estimation $\left\|\tau^{h}-4 / 3 \tau_{h}^{H}\right\|_{2}$ for all conservative equations, both for a uniform distribution of nodes and for a smooth non-uniform distribution (governed by Eq. (30)), is plotted in Fig. 17(a)-(b) for quads-based grids and Fig. 17(c)-(d) for triangles-based grids. As predicted by Eq. (8), the estimation is not accurate when second-order interpolation is used to restrict the solution. The error in the estimation has the same magnitude as the exact truncation error. An interpolation of order $s>p$ is required. When the third order is used to restrict the solution from a fine to a coarse grid, the error in the estimation decreases with a rate of convergence higher than two when the grid is refined, which ensures that the estimation tends to be accurate when using a second-order spatial scheme. It must be noted that in this particular case, the use of a higher order restriction operator (here, injection) does not increase the accuracy of the estimation; thus, the 
(a)

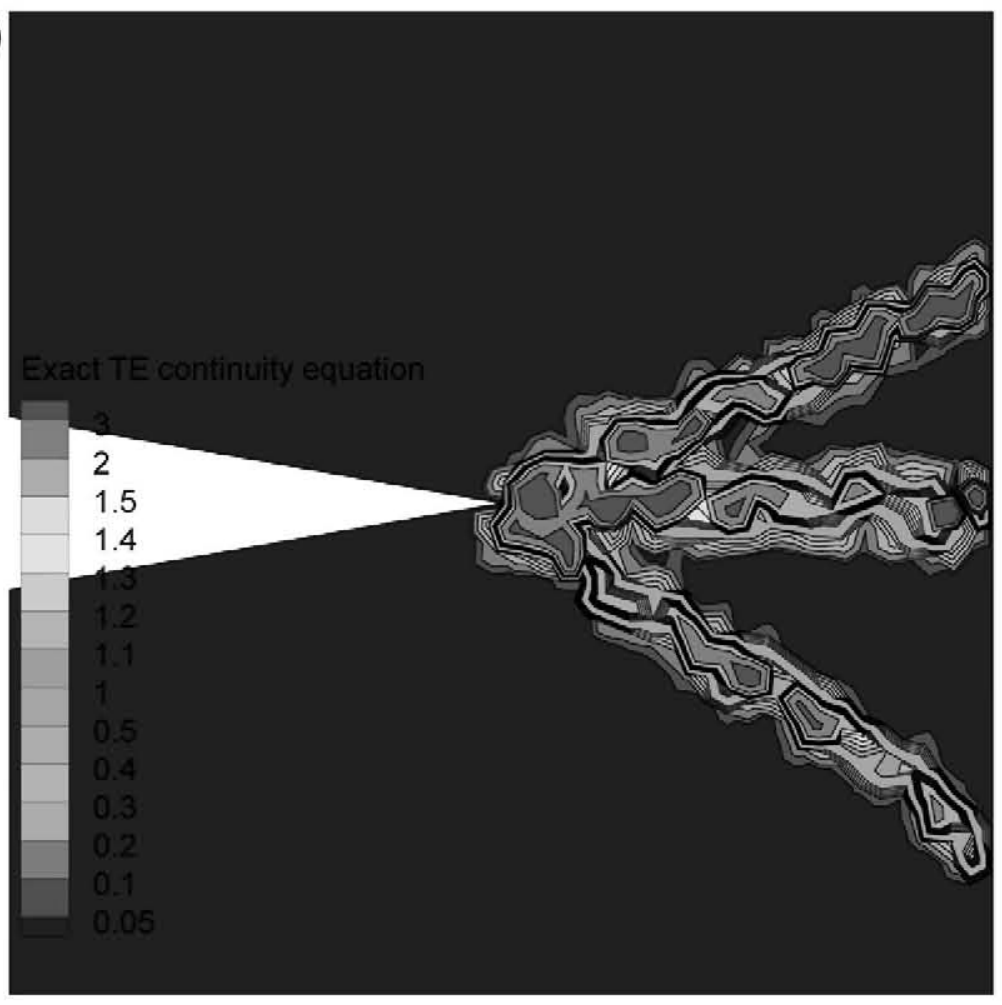

(b)

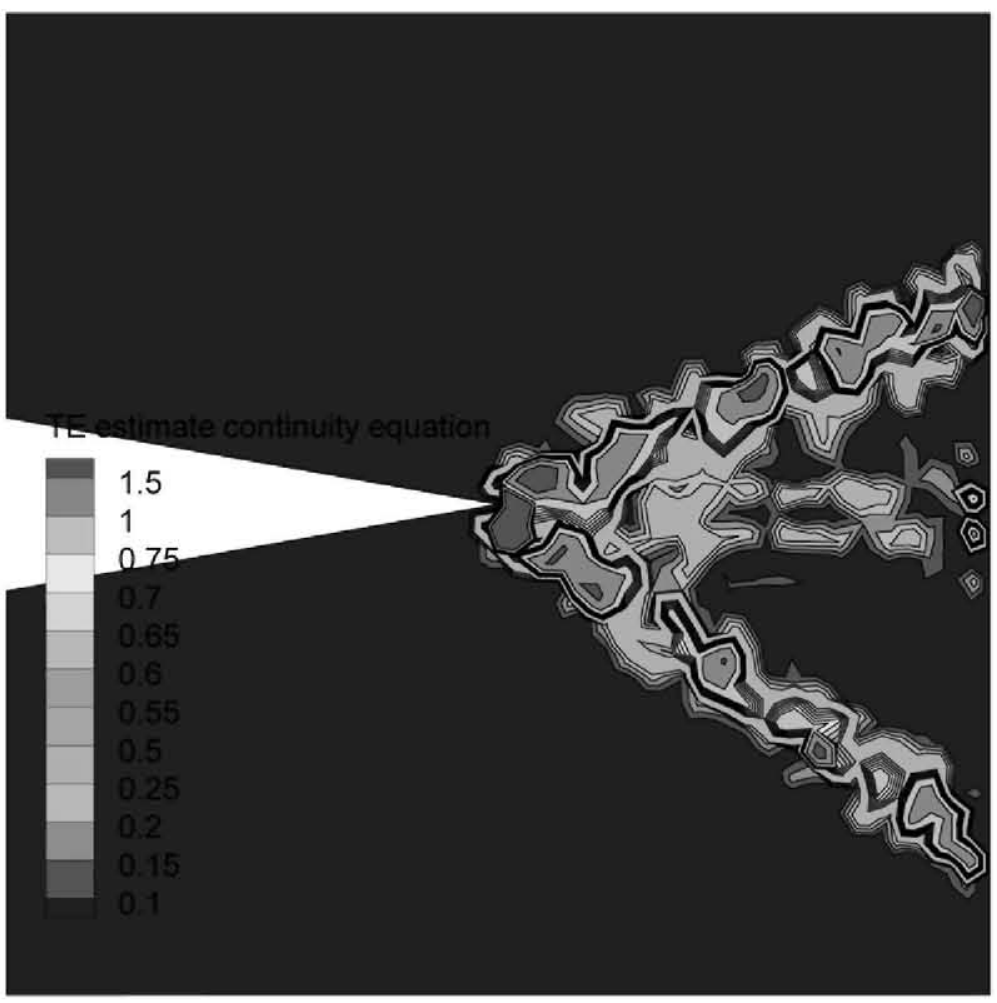

Fig. 20. Supersonic confluence flow. (a) Exact truncation error and (b) truncation error estimate.

following term in the Taylor series of the truncation error does not vanish, as is the case for the Laplacian operator (using a centred FD stencil). 
As mentioned in the previous section, it is of importance to maintain some consistency in the topology of the mesh between the fine grid and the coarse grid. To observe the effect of the element type on the shape of the truncation error, we performed a computation using a $129 \times 129$ uniform destructured grid. We computed the exact truncation error on this current fine grid and on a coarse grid obtained by the agglomeration of fine grid cells (see Fig. 18(a)-(b)). In this specific case, the agglomeration algorithm generates a diagonal that crosses all of the domain in which the element orientations change. The contours of the exact truncation errors are reported in Fig. 18(c)-(d). In Fig. 18(c)-(d), the absolute value of the exact truncation error is depicted for the continuity equation for the fine grid and the agglomerated grid. It is illustrated in these plots that the shape and magnitude of the truncation error vary significantly between these grids, and high values are located where the orientation of the elements change; then, the analysis of Section 2 does not hold, as the error does not linearly decrease with the mesh spacing. Going back to Eq. (38), it is clear that the truncation error depends on the orientation of the faces, yielding a different distribution when computed on different elements or elements with a different orientation. Then, if the truncation error is to be estimated, it is mandatory that the fine grid and the coarse grid possess the same type of elements.

\subsection{Supersonic confluent flow}

Many fluid dynamics problems involve transonic flows, eventually with shocks. The methodology presented here to estimate the truncation error makes assumptions on the smoothness of the flow, as it is based on the existence of a development of the solution in the Taylor series. However, it is of interest to examine the behaviour of the estimator in the presence of discontinuities. For this purpose, two-dimensional Euler computations were performed on the supersonic confluent flow. This test case is a pure supersonic flow with two shocks and a slip line. The geometry of the problem is schematically illustrated in Fig. 19(a). In zones 3 and 4 , the pressure is $p_{3}=p_{4}=1.7238$, and the slip line between zones 3 and 4 creates an angle of $-0.745^{\circ}$ with the horizontal direction. The oblique shock wave angles are $\beta_{3}=33.605^{\circ}$ and $\beta_{4}=44.992^{\circ}$. All flow variables can be computed using the oblique shock wave theory.

The truncation error estimation was performed on a fully unstructured grid composed of 3558 nodes and 3346 triangles (see Fig. 19(b)), whereas the fine grid (where the flow was computed) was obtained by uniform refinement (edge bisection). The generation of the grids is performed in this manner to maintain some consistency in the quality and also to allow the use of the injection operator. As the flow exhibits strong shocks and a slip line, the assumptions of smoothness stated in Section 2 do not hold; thus, no attempt was made to perform a rigorous analysis of the accuracy of the truncation error estimator. A qualitative analysis is more adequate in this case. The exact truncation error and the estimation of the truncation error are reported in Fig. 20(a)-(b). The truncation error estimator correctly predicts the locations of the error, mainly at the shocks. The slip line, where the velocity magnitude is discontinuous, is also predicted but with less precision. The extension to vectorial equations does not pose any problem to accurately estimate the truncation error. Although most of the work reported in the literature was performed using structured solvers, the extension of this method to arbitrary elements is also possible as long as the fine and coarse grids share the same topology.

\section{Conclusions}

Accurate estimations of the local truncation error have been successfully performed on FD solvers and have been extended to FV solvers on uniform, smooth non-uniform and distorted grids. Conditions on the order of accuracy of the restriction operators, the choice of the boundary conditions, the distortion of the grids and the magnitude of the iteration error to ensure accurate estimations have been derived and verified numerically on the scalar Poisson equation and on Euler equations. In this approach, a converged solution is not assumed; thus, an analysis of the accuracy of the estimation has been performed within the iteration process to the steady state. The results demonstrated that if the restriction operators and/ or the coarse grid discretisation are chosen carefully, then the estimation is accurate at the first iteration and yields a robust a priori error estimator. If no special attention is provided, then the estimation is accurate as long as the magnitude of the iteration error remains lower than the truncation error. The estimation of the truncation error presented here is based on the smoothness of the flow; however, when shocks are considered, the estimator behaves well and predicts the regions of high errors. In this paper, we extended the method to arbitrary elements and demonstrated that accurate estimations are also possible using any type of element with the necessary condition that the elements used for the fine and coarse grids are the same. With an accurate estimation of the local truncation error in hand, several applications are natural, such as mesh generation and mesh adaptation, higher order reconstruction or zonal modelling.

\section{Acknowledgements}

This work has been supported by the Spanish Ministry of Industry under grant TSI-020100-2010-827.

\section{References}

[1] W.L. Oberkampf, C.J. Roy, Verification and Validation in Scientific Computing, Cambridge University Press, Cambridge, 2010.

[2] P.J. Roache, Verification and Validation in Computational Science and Engineering, Hermosa Publishers, Albuquerque, NM, 1998. 
[3] C.]. Roy, Review of discretization error estimators in scientific computing, AIAA Paper, 2010-126.

[4] W. Briggs, V.E. Henson, S.S. McCormick, A Multigrid Tutoriala, 2nd ed., Society for Industrial and Applied Mathematics, Philadelphia, PA, 2000.

[5] U. Trottenberg, C. Oosterlee, A. Schler, Multigrid, Academic Press.

[6] A. Brandt, Multigrid techniques: 1984 Guide with applications to fluid dynamics.

[7] C. Ilinca, X.D. Zhang, J-Y. Trpanier, R. Camarero, A comparison of three error estimation techniques for finite-volume solutions of compressible flows, Comput. Methods Appl. Mech. Eng. 189 (2000) 1277-1294.

[8] M. Garbey, W. Shyy, Error estimation, multilevel method and robust extrapolation in the numerical solution of PDEs, in: 14th International Conference on Domain Decomposition Methods.

[9] T.S. Phillips, C. Roy, Evaluation of extrapolation-based discretization error and uncertainty estimators, in: 49th AIAA Aerospace Science Meeting, AIAA 2011-215.

[10] T. I-P. Shih, B.R. Williams, Development and evaluation of an a posteriori method for estimating and correcting grid-induced errors in solutions of the Navier-Stokes equations, in: 47th AIAA Aerospace Science Meeting, AIAA 2009-1499.

[11] M.B. Giles, N.A. Pierce, Adjoint equations in CFD: duality, boundary conditions and solution behaviour, AIAA Paper, 97-1850.

[12] D.A. Venditti, D.L. Darmofal, Adjoint Error Estimation and Grid Adaptation for Functional Outputs: Application to Quasi-One- Dimensional Flow, J. Comput. Phys. 164 (2000) 204-227.

[13] Relationship between the Truncation Errors of Centered Finite-Difference Approximations on Uniform and Nonuniform Meshes, Note, Journal of Computational Physics, 46, 1982, pp. 469-474.

[14] Leonard, B.P., Comparison of Truncation Error of Finite-Difference and Finite-Volume Formulations of Convection Terms, NASA Technical Memorandum 105861, ICOMP-92-19, September 1992.

[15] Y.N. Jeng, J.L. Chen, Truncation Error Analysis of the Finite Volume Method for Steady Convective Equation, J. Comput. Phys. 100 (1992) 64-76.

[16] S.F. Hagen, Estimation of the Truncation Error for the Linearized, Shallow Water Momentum Equations, Eng. Comput. 17 (2001) 354-362.

[17] Y. Kallinderis, C. Kontzialis, A priori mesh quality estimation via direct relation between truncation error and mesh distortion, J. Comput. Phys. 228 (2009) 881-902.

[18] Berger, M.]., Adaptive Finite Difference Methods in Fluid Dynamics, Mathematics and Computers, February 1987.

[19] K. Bernert, $\tau$-Extrapolation-Theoretical foundation, numerical experiment, and application to Navier-Stokes equations, SIAM J. Sci. Comput. 18 (2) (1997) 460-478.

[20] S.R. Fulton, On the accuracy of multigrid truncation error estimates, Electron. Trans. Numer. Anal. 15 (2003) $29-37$.

[21] A. Syrakos, A. Goulas, Estimate of the truncation error of finite volume discretization of the Navier-Stokes equations on colocated grids, Int. J. Numer. Methods Fluids 50 (2006) 103-130.

[22] T. Gerhold, V. Hannemann, D. Schwamborn, On the validation of the DLR-TAU Code, new results in numerical and experimental fluid mechanics, Notes Numer. Fluid Mech. 72 (1999) 426-433.

[23] P. Wesseling, An introduction to multigrid methods, John Wiley \& Sons, 1991

[24] C.J. Roy, Review of code and solution verification procedures for computational simulation, J. Comput. Phys. 205 (2005) $131-156$.

[25] Y. Wada, A.V. Johansson, A flux splitting scheme with high resolution and robustness for discontinuities, AIAA Paper, 94-0083. 